\title{
Refined asymptotics for the blow-up solution of the complex Ginzburg-Landau equation in the subcritical case
}

\author{
Giao Ky Duong, Nejla Nouaili, and Hatem Zaag
}

\begin{abstract}
In this paper, we aim to refine the blow-up behavior for the complex Ginzburg-Landau (CGL) equation in some subcritical case. More precisely, we construct blow-up solutions and refine their blow-up profile to the next order.
\end{abstract}

\section{Introduction}

In this paper, we consider the complex Ginzburg-Landau (CGL) equation

$$
\begin{aligned}
& u_{t}=(1+i \beta) \Delta u+(1+i \delta)|u|^{p-1} u+\alpha u, \\
& u(., 0)=u_{0} \in L^{\infty}\left(\mathbb{R}^{N}, \mathbb{C}\right),
\end{aligned}
$$

where $\delta, \beta, \alpha \in \mathbb{R}$.

This equation is better known when $p=3$, having a long history in physics (see Aranson and Kramer [2]). The CGL equation describes many phenomena including nonlinear waves, second-order phase transitions, and superconductivity. We note that the CGL equation can be used to describe the evolution of amplitudes of unstable modes for any process exhibiting a Hopf bifurcation (see for example [2, Sect. VI-C, p. 37; Sect. VII, p. 40] and the references cited therein). In addition, our equation can be considered as a general normal form for a large class of bifurcations and nonlinear wave phenomena in continuous media systems. More generally, the CGL equation is used to describe synchronization and collective oscillation in complex media.

The study of collapse, chaotic or blow-up solutions of equation (CGL) appears in many works; for a description of an unstable plane Poiseuille flow, see Stewartson and Stuart [35] and Hocking et al. [21] and, in the context of binary mixtures, see Kolodner et al. $[22,23]$, where the authors describe an extensive series of experiments on traveling-wave convection in an ethanol/water mixture, and they observe collapse solutions that appear experimentally. For blow-up phenomena, see Plecháč and Šverák [29] and Rottschäfer $[33,34]$.

2020 Mathematics Subject Classification. 35K57, 35K40, 35B44.

Keywords. Blow-up profile, complex Ginzburg-Landau equation. 
For our purpose, we consider CGL independently from any particular physical context and investigate it as a mathematical model in partial differential equations with $p>1$.

The Cauchy problem for equation (CGL) can be solved in a variety of spaces using the semigroup theory as in the case of the heat equation (see $[5,18,19])$. The space $L^{\infty}\left(\mathbb{R}^{N}\right)$ is a convenient choice for us.

We say that $u(t)$ blows up or collapses in finite time $T<\infty$, if $u(t)$ exists for all $t \in[0, T)$ and $\lim _{t \rightarrow T}\|u(t)\|_{L^{\infty}}=+\infty$. In that case, $T$ is called the blow-up time of the solution. A point $x_{0} \in \mathbb{R}^{N}$ is said to be a blow-up point if there is a sequence $\left\{\left(x_{j}, t_{j}\right)\right\}$, such that $x_{j} \rightarrow x_{0}, t_{j} \rightarrow T$ and $\left|u\left(x_{j}, t_{j}\right)\right| \rightarrow \infty$ as $j \rightarrow \infty$. The set of all blow-up points is called the blow-up set.

Let us now introduce the following definition:

Definition 1.1 (Criticality for CGL). The parameters $(\beta, \delta)$ are in the subcritical (resp. critical, supercritical) regime if $p-\delta^{2}-\beta \delta(p+1)$ is positive (resp. zero, negative).

Some results are available in the subcritical regime from Zaag [38] $(\beta=0)$ and also Masmoudi and Zaag [24] $(\beta \neq 0)$. In those papers, the authors construct a solution of equation (CGL), which blows up in finite time $T>0$ only at the origin such that for all $t \in[0, T)$,

$$
\begin{aligned}
& \left\|(T-t)^{\frac{1+i \delta}{p-1}}|\log (T-t)|^{-i \mu} u(x, t)-\left(p-1+\frac{b_{\text {sub }}|x|^{2}}{(T-t)|\log (T-t)|}\right)^{-\frac{1+i \delta}{p-1}}\right\|_{L^{\infty}} \\
& \leq \frac{C_{0}}{1+\sqrt{|\log (T-t)|}}
\end{aligned}
$$

where

$$
b_{\text {sub }}=\frac{(p-1)^{2}}{4\left(p-\delta^{2}-\beta \delta(1+p)\right)}>0 \quad \text { and } \quad \mu=-\frac{2 b_{\text {sub }}}{(p-1)^{2}} \beta\left(1+\delta^{2}\right) .
$$

Note that this result was previously obtained formally by Hocking and Stewartson [20] $(p=3)$ and mentioned later in Popp et al. [30] (see those references for more blow-up results often proved numerically, in various regimes of the parameters).

In the critical regime, some blow-up solutions are available from Nouaili and Zaag [28] and also Duong, Nouaili and Zaag [10]. More precisely, in that regime, the authors construct a solution of equation (CGL), which blows up in finite time $T>0$ only at the origin, such that for all $t \in[0, T)$ (see [10, Thm. 2]),

$$
\begin{aligned}
& \|(T-t)^{\frac{1+i \delta}{p-1}}|\log (T-t)|^{-i \mu} e^{-i v \sqrt{|\log (T-t)|}} u(x, t) \\
& -\left(p-1+\frac{b_{\text {cri }}|x|^{2}}{(T-t)|\log (T-t)|^{1 / 2}}\right)^{-\frac{1+i \delta}{p-1}} \|_{L^{\infty}} \leq \frac{C_{0}}{1+|\log (T-t)|^{1 / 4}},
\end{aligned}
$$

where

$$
b_{\mathrm{cri}}^{2}=\frac{(p-1)^{2} 4(p+1)^{2} \delta^{2}}{16\left(1+\delta^{2}\right)\left(p(2 p-1)-(p-2) \delta^{2}\right)\left((p+3) \delta^{2}+p(3 p+1)\right)}>0
$$


and $v=v(\beta, p), \mu=\mu(\beta, p)$ are given in [10]. In fact, the authors obtain a more refined description showing some additional higher-order terms in the Taylor expansion of the blow-up solution given in (1).

Following our result, we felt that no similar refinement exists in the subcritical regime, except maybe for some formal results given by Berger and Kohn [3] and also Velázquez, Galaktionov and Herrero [37] when $\beta=\delta=\alpha=0$, which corresponds to the nonlinear heat equation (NLH).

\subsection{Statement of our result}

Our main concern is to give a refined asymptotic description of the blow-up solution given by Masmoudi and Zaag [24]. Rather than considering that solution and refining its description, we will instead start over from the beginning, and construct a solution $u(x, t)$ of (CGL) in the subcritical regime $\left(p-\beta \delta(p+1)-\delta^{2}>0\right)$ that blows up in some finite time $T$, in the sense that

$$
\lim _{t \rightarrow T}\|u(., t)\|_{L^{\infty}}=+\infty,
$$

and which has the same zero-order description as the solution of Masmoudi and Zaag [24] given in (1), with a more accurate description showing the next order terms in the expansion.

We consider $u(x, t)$ a solution of (CGL). Let us first introduce self-similar variables

$$
W(y, t)=(T-t)^{\frac{1+i \delta}{p-1}} u(x, t), \quad y=\frac{x}{\sqrt{T-t}} ;
$$

then, the main result of this work is the following:

Theorem 1 (First-order terms). Let us consider the subcritical regime where $p-\delta^{2}-$ $\beta \delta(p+1)>0$. Then there exists a unique constant $\eta$ depending on $p, \delta$ and $\beta$ such that equation (CGL) has a solution $u(x, t)$, which blows up in finite time $T$, only at the origin. Moreover, the solution decomposes in self-similar variables as follows: for $M>0$,

$$
\begin{aligned}
\sup _{|y|<M|\log (T-t)|^{\frac{1}{2}}} & \left.\left|W(y, t) e^{-i \eta \frac{\log (|\log (T-t)|)}{|\log (T-t)|}}\right| \log (T-t)\right|^{-i \mu} e^{i \theta(t)} \\
& -\left\{\varphi_{0}\left(\frac{y}{|\log (T-t)|^{1 / 2}}\right)+\frac{a(1+i \delta)}{|\log (T-t)|}+\frac{\log |\log (T-t)|}{|\log (T-t)|^{2}} \mathcal{E}(y)\right. \\
& \left.+\frac{1}{|\log (T-t)|^{2}} \mathcal{F}(y)\right\} \mid \\
\leq & C(M) \frac{|\log | \log (T-t)||^{2}}{|\log (T-t)|^{3}}\left(1+|y|^{5}\right),
\end{aligned}
$$

and $\theta(t) \rightarrow \theta_{0}$ as $t \rightarrow T$, such that

$$
\left|\theta(t)-\theta_{0}\right| \leq C \frac{\mid \log \left(\left.|\log (T-t)|\right|^{2}\right.}{|\log (T-t)|^{2}}
$$


with

$$
\varphi_{0}(z)=\left(p-1+b z^{2}\right)^{-\frac{1+i \delta}{p-1}},
$$

with $b\left(=b_{\text {sub }}\right)$ defined as in (2),

$$
\mu=-\frac{4 b \beta\left(1+\delta^{2}\right)}{(p-1)^{2}}, \quad a=2 \kappa(1-\beta \delta) \frac{b}{(p-1)^{2}} \quad \text { and } \quad \kappa=(p-1)^{-\frac{1}{p-1}} .
$$

The functions $\mathcal{E}(y)$ and $\mathcal{F}(y)$ are defined as

$$
\begin{aligned}
\mathcal{E}(y) & =\tilde{\mathcal{A}}_{2} \tilde{h}_{2}(y), \\
\mathcal{F}(y) & =\widetilde{\mathcal{B}}_{0} \tilde{h}_{0}(y)+\mathscr{B}_{2} h_{2}(y)+\widetilde{\mathcal{B}}_{2} \tilde{h}_{2}(y),
\end{aligned}
$$

where $\widetilde{\mathcal{B}}_{0}, \mathscr{B}_{2}$ and $\widetilde{\mathcal{A}}_{2}$ depend only on $\beta$ and $\delta$ and are given by (41) in Definition 3.1 and $h_{0}(y), h_{2}(y)$ and $\tilde{h}_{2}(y)$ will be given in Lemma 2.2 .

The constant $\widetilde{\mathcal{B}}_{2}$ depends only on $\beta$ and $\delta$ when $\beta \neq 0$. When $\beta=0$, we can choose $\widetilde{\mathcal{B}}_{2}$ arbitrarily.

Remark 1.2. For technical reasons, the proof of Theorem 1 must be done separately for $\beta \neq 0$ and $\beta=0$.

Remark 1.3. In the case of the nonlinear heat equation $(\beta=\delta=0)$, Theorem 1 presents the first rigorous proof of the formal approach given by Velázquez, Galaktionov, and Herrero [37].

Remark 1.4. We will consider CGL, given by (CGL), only when $\alpha=0$. The case $\alpha \neq 0$ can be done as in [12]. In fact, when $\alpha \neq 0$, exponentially small terms will be added to our estimates in a self-similar variable (see (9) below), and that will be absorbed in our error terms, since our trap $\mathcal{V}_{A}(s)$ defined in Definition 3.1 is given in polynomial scales.

Let us give an idea of the method used to prove the results. We construct the blow-up solution with the profile in Theorem 1 , by following the method of [4, 25]. This kind of method has been applied to various nonlinear evolution equations. For hyperbolic equations, it has been successfully used for the construction of multi-solitons for the semilinear wave equation in one space dimension (see [6]). For parabolic equations, it has been used in [24] and [39] for the complex Ginzburg Landau (CGL) equation with no gradient structure, the critical harmonic heat flow in [31], the two-dimensional Keller-Segel equation in [32] and the nonlinear heat equation involving a nonlinear gradient term in $[12,36]$. Recently, this method has been applied to various nonvariational parabolic systems in [27] and [13-16], and to a logarithmically perturbed nonlinear equation in [7-9,26]. We also mention a result for a higher-order parabolic equation [17] and in [1,11] two more results for equations involving nonlocal terms.

Following [25], [28] and [10], the proof is divided into two steps. First, we reduce the problem to a finite-dimensional case. Second, we solve the finite-time-dimensional problem and conclude by contradiction using index theory. More precisely, the proof is performed in the framework of the similarity variables defined below in (9). We linearize 
the self-similar solution around the profile $\varphi_{0}$ and we obtain $q$ (see (11) below). Our goal is to guarantee that $q(s)$ belongs to some set $\mathcal{V}_{A}(s)$ (introduced in Definition 3.1), which shrinks to 0 as $s \rightarrow+\infty$. The proof relies on two arguments:

- The linearized equation gives two positives modes $\left(\tilde{Q}_{0}\right.$ and $\left.\tilde{q}_{1}\right)$, one zero mode $\left(\tilde{q}_{2}\right)$ and an infinite-dimensional negative part. The negative part is easily controlled by the effect of the heat kernel. Control of the zero mode is quite delicate. The control of $q$ is reduced to control of its positive modes; see Proposition 3.8.

- Control of the positive modes $\widetilde{Q}_{0}$ and $\tilde{q}_{1}$ is handled thanks to a topological argument based on index theory; see Proposition 3.5.

The organization of the rest of this paper is as follows. Section 3, the heart of the paper, is divided into three subsections. At the beginning of Section 3 we give the proof of the existence of the profile assuming technical details when $\beta \neq 0$. In particular, we construct a shrinking set and give an example of initial data giving rise to the blow-up profile. Section 3.1 is devoted to the proof of technical results which are needed in the proof of existence; see Proposition 3.8. Then in Section 3.2 we aim to give an a priori estimate of the finite mode of $q$ the negative part $q_{-}$and the outer part $q_{e}$. In Section 3.3, we explain the case $\beta=0$. In addition to that, we also give Appendices $\mathrm{A}, \mathrm{B}, \mathrm{C}$, which give necessary computations in detail and give some fundamental estimates used for the proofs in the paper.

\section{Formulation of the problem}

Here we consider the CGL equation, introduced in (CGL), with $\alpha=0$. As we mentioned before in Remark 1.4, the perturbation of $\alpha u$ is quite small. Now let us introduce the similarity variable

$$
u(x, t)=(T-t)^{-\frac{1}{p-1}} w(y, s), \quad y=\frac{x}{\sqrt{T-t}} \quad \text { and } \quad s=-\ln (T-t) .
$$

Hence, $w$ reads

$$
\partial_{s} w=(1+i \beta) \Delta w-\frac{1}{2} y \cdot \nabla w-\frac{w}{p-1}+(1+i \delta)|w|^{p-1} w .
$$

Using the idea from [24], we will introduce $q(y, s)$ and $\theta(s)$ satisfying

$$
w(y, s)=e^{i\left(\mu \ln s+\eta \frac{\ln s}{s}+\theta(s)\right)}(\varphi(y, s)+q(y, s)),
$$

where

$$
\varphi(y, s)=\varphi_{0}\left(\frac{y}{s^{1 / 2}}\right)+(1+i \delta) \frac{a}{s} \equiv \kappa^{-i \delta}\left(p-1+b \frac{|y|^{2}}{s}\right)^{-\frac{1+i \delta}{p-1}}+(1+i \delta) \frac{a}{s},
$$

where $\mu, a$ and $b$ are well known in [24]:

$$
\mu=-\frac{2 b \beta\left(1+\delta^{2}\right)}{(p-1)^{2}}, \quad a=2 \kappa(1-\beta \delta) \frac{b}{(p-1)^{2}},
$$


and

$$
b=\frac{(p-1)^{2}}{4\left(p-\delta^{2}-(p+1) \delta \beta\right)} .
$$

We will explain how we choose these constants in the proof. In particular, $\eta$ is a new constant added for refinement of the behavior of $w$. Note that $\varphi_{0}(z)$ satisfies

$$
-\frac{1}{2} z \cdot \nabla \varphi_{0}-\frac{1+i \delta}{p-1} \varphi_{0}+(1+i \delta)\left|\varphi_{0}\right|^{p-1} \varphi_{0}=0 .
$$

Using equation (10), we derive that $q$ solves the equation

$$
\frac{\partial q}{\partial s}=\mathscr{L}_{\beta} q-\frac{(1+i \delta)}{p-1} q+L\left(q, \theta^{\prime}, y, s\right)+R^{*}\left(\theta^{\prime}, y, s\right),
$$

where

$$
\begin{aligned}
& \mathscr{L}_{\beta} q=(1+i \beta) \Delta q-\frac{1}{2} y \cdot \nabla q, \\
& \begin{aligned}
& L\left(q, \theta^{\prime}, y, s\right)=(1+i \delta)\left\{|\varphi+q|^{p-1}(\varphi+q)-|\varphi|^{p-1} \varphi\right. \\
&\left.\quad-i\left(\eta\left(\frac{1}{s^{2}}-\frac{\ln s}{s^{2}}\right)+\frac{\mu}{s}+\theta^{\prime}(s)\right) q\right\},
\end{aligned} \\
& R^{*}\left(\theta^{\prime}, y, s\right)=R(y, s)-i\left(\eta\left(\frac{1}{s^{2}}-\frac{\ln s}{s^{2}}\right)+\frac{\mu}{s}+\theta^{\prime}(s)\right) \varphi, \\
& R(y, s)=-\frac{\partial \varphi}{\partial s}+(1+i \beta) \Delta \varphi-\frac{1}{2} y \cdot \nabla \varphi-\frac{(1+i \delta)}{p-1} \varphi+(1+i \delta)|\varphi|^{p-1} \varphi .
\end{aligned}
$$

Our aim is to find a $\theta \in C^{1}([-\ln T, \infty), \mathbb{R})$ such that equation (18) has a solution $q(y, s)$ defined for all $(y, s) \in \mathbb{R}^{N} \times[-\ln T, \infty)$ such that

$$
q(y, s)=\frac{\mathcal{F}(y) \log s}{s^{2}}+v(y, s)
$$

where $\mathcal{F}$ is defined by (8) in Theorem 1 and

$$
\|v(s)\|_{L^{\infty}} \rightarrow 0 \quad \text { as } s \rightarrow \infty
$$

From (13), one sees that the variable $z=\frac{y}{s^{1 / 2}}$ plays a fundamental role. Thus, we will consider the dynamics for $|z|>K$, and $|z|<2 K$ separately for some $K>0$ to be fixed large.

\subsection{The outer region where $|y|>K s^{1 / 2}$}

Let us consider a nonincreasing cut-off function $\chi_{0} \in C^{\infty}\left(\mathbb{R}^{+},[0,1]\right)$ such that $\chi_{0}(\xi)=1$ for $\xi<1$ and $\chi_{0}(\xi)=0$ for $\xi>2$ and introduce

$$
\chi(y, s)=\chi_{0}\left(\frac{|y|}{K s^{1 / 2}}\right),
$$


where $K$ will be fixed large. Let us define

$$
q_{e}(y, s)=e^{\frac{i \delta}{p-1} s} q(y, s)(1-\chi(y, s)),
$$

and note that $q_{e}$ is the part of $q(y, s)$, corresponding to the non-blow-up region $|y|>$ $K s^{1 / 2}$. As we will explain in Section 3.2.3, the linear operator of the equation satisfied by $q_{e}$ is negative, which makes it easy to control $\left\|q_{e}(s)\right\|_{L^{\infty}}$. This is not the case for the part of $q(y, s)$ for $|y|<2 K s^{1 / 2}$, where the linear operator has two positive eigenvalues, a zero eigenvalue in addition to infinitely many negative ones. Therefore, we have to expand $q$ with respect to these eigenvalues in order to control $\|q(s)\|_{L^{\infty}\left(|y|<2 K s^{1 / 2}\right)}$. This requires more work than for $q_{e}$. The following subsection is dedicated to that purpose. From now on, $K$ will be a fixed constant which is chosen such that $\left\|\varphi\left(s^{\prime}\right)\right\|_{L^{\infty}\left(|y|>K s^{\prime 1 / 2}\right)}$ is small enough, namely $\left\|\varphi_{0}(z)\right\|_{L^{\infty}(|z|>K)}^{p-1} \leq \frac{1}{C(p-1)}$ (see Section 3.2.3 below for more details).

\subsection{The inner region where $|y|<2 K s^{1 / 2}$}

If we linearize the term $L\left(q, \theta^{\prime}, y, s\right)$ in equation (14), then we can write (14) as

$$
\begin{aligned}
\frac{\partial q}{\partial s}= & \mathscr{L}_{\beta, \delta} q-i\left(\frac{\mu}{s}-\eta \frac{\ln s}{s^{2}}+\frac{\eta}{s^{2}}+\theta^{\prime}(s)\right) q \\
& +V_{1} q+V_{2} \bar{q}+B(q, y, s)+R^{*}\left(\theta^{\prime}, y, s\right),
\end{aligned}
$$

where

$$
\begin{aligned}
& \mathscr{L}_{\delta, \beta} q=(1+i \beta) \Delta q-\frac{1}{2} y \cdot \nabla q+(1+i \delta) \operatorname{Re} q, \\
& V_{1}(y, s)=(1+i \delta) \frac{p+1}{2}\left(|\varphi|^{p-1}-\frac{1}{p-1}\right), \\
& V_{2}(y, s)=(1+i \delta) \frac{p-1}{2}\left(|\varphi|^{p-3} \varphi^{2}-\frac{1}{p-1}\right), \\
& B(q, y, s)=(1+i \delta)\left(|\varphi+q|^{p-1}(\varphi+q)-|\varphi|^{p-1} \varphi-|\varphi|^{p-1} q\right. \\
& \left.\quad-\frac{p-1}{2}|\varphi|^{p-3} \varphi(\varphi \bar{q}+\bar{\varphi} q)\right), \\
& R^{*}\left(\theta^{\prime}, y, s\right)=R(y, s)-i\left(\frac{\mu}{s}-\eta \frac{\ln s}{s^{2}}+\frac{\eta}{s^{2}}+\theta^{\prime}(s)\right) \varphi, \\
& R(y, s)=-\frac{\partial \varphi}{\partial s}+\Delta \varphi-\frac{1}{2} y \cdot \nabla \varphi-\frac{(1+i \delta)}{p-1} \varphi+(1+i \delta)|\varphi|^{p-1} \varphi .
\end{aligned}
$$

Note that the term $B(q, y, s)$ is built to be quadratic in the inner region $|y| \leq K s^{\frac{1}{2}}$. Indeed, we have for all $K \geq 1$ and $s \geq 1$,

$$
\sup _{|y| \leq 2 K s^{\frac{1}{2}}}|B(q, y, s)| \leq C(K)|q|^{2} .
$$

Note also that $R(y, s)$ measures the defect of $\varphi(y, s)$ from being an exact solution of (10). However, since $\varphi(y, s)$ is an approximate solution of $(10)$, one easily derives the fact that

$$
\|R(s)\|_{L^{\infty}} \leq \frac{C}{s}
$$


Therefore, if $\theta^{\prime}(s)$ goes to zero as $s \rightarrow \infty$, we expect the term $R^{*}\left(\theta^{\prime}, y, s\right)$ to be small, since (18) and (21) yield

$$
\left|R^{*}\left(\theta^{\prime}, y, s\right)\right| \leq \frac{C}{s}+\left|\theta^{\prime}(s)\right|
$$

Therefore, since we would like to make $q$ go to zero as $s \rightarrow \infty$, the dynamics of equation (18) are influenced by the asymptotic limit of its linear term,

$$
\mathscr{L}_{\beta, \delta} q+V_{1} q+V_{2} \bar{q},
$$

as $s \rightarrow \infty$. In the sense of distributions (see the definitions of $V_{1}$ and $V_{2}$ in (18) and $\varphi$ in (12)) this limit is $\mathscr{L}_{\beta, \delta} q$.

\subsection{Spectral properties of $\mathscr{L}_{\beta}$}

Here we will restrict to $N=1$. We consider the Hilbert space $L_{\left|\rho_{\beta}\right|}^{2}\left(\mathbb{R}^{N}, \mathbb{C}\right)$ which is the set of all $f \in L_{\text {loc }}^{2}\left(\mathbb{R}^{N}, \mathbb{C}\right)$ such that

$$
\int_{\mathbb{R}^{N}}|f(y)|^{2}\left|\rho_{\beta}(y)\right| d y<+\infty,
$$

where

$$
\rho_{\beta}(y)=\frac{e^{-\frac{|y|^{2}}{4(1+i \beta)}}}{(4 \pi(1+i \beta))^{N / 2}} \quad \text { and } \quad\left|\rho_{\beta}(y)\right|=\frac{e^{-\frac{|y|^{2}}{4\left(1+\beta^{2}\right)}}}{\left(4 \pi \sqrt{1+\beta^{2}}\right)^{N / 2}} .
$$

We can diagonalize $\mathscr{L}_{\beta}$ in $L_{\left|\rho_{\beta}\right|}^{2}\left(\mathbb{R}^{N}, \mathbb{C}\right)$. Indeed, we can write

$$
\mathscr{L}_{\beta} q=\frac{1}{\rho_{\beta}} \operatorname{div}\left(\rho_{\beta} \nabla q\right) .
$$

We notice that $\mathscr{L}_{\beta}$ is formally "self-adjoint" with respect to the weight $\rho_{\beta}$. Indeed, for any $v$ and $w$ in $L_{\left|\rho_{\beta}\right|}^{2}\left(\mathbb{R}^{N}, \mathbb{C}\right)$ satisfying $\mathscr{L}_{\beta} v$ and $\mathscr{L}_{\beta} w$ in $L_{\left|\rho_{\beta}\right|}^{2}\left(\mathbb{R}^{N}, \mathbb{C}\right)$, it holds that

$$
\int v \mathscr{L}_{\beta} w \rho_{\beta} d y=\int w \mathscr{L}_{\beta} v \rho_{\beta} d y .
$$

If we introduce for each $\alpha=\left(\alpha_{1}, \ldots, \alpha_{N}\right) \in \mathbb{N}^{N}$ the polynomial

$$
f_{\alpha}(y)=c_{\alpha} \Pi_{i=1}^{N} H_{\alpha_{i}}\left(\frac{y_{i}}{2 \sqrt{1+i \beta}}\right)
$$

where $H_{n}$ is the standard one-dimensional Hermite polynomial and $c_{\alpha} \in \mathbb{C}$ is chosen so that the term of highest degree in $f_{\alpha}$ is $\Pi_{i=1}^{N} y_{i}^{\alpha_{i}}$, then we get a family of eigenfunctions of $\mathscr{L}_{\beta}$, "orthogonal" with respect to the weight $\rho_{\beta}$, in the sense that for any different $\alpha$ and $\sigma \in \mathbb{N}^{N}$,

$$
\begin{gathered}
\mathscr{L}_{\beta} f_{\alpha}=-\frac{\alpha}{2} f_{\alpha}, \\
\int_{\mathbb{R}} f_{\alpha}(y) f_{\sigma}(y) \rho_{\beta}(y) d y=0 .
\end{gathered}
$$




\subsection{Spectral properties of $\mathscr{L}_{\beta, \delta}$}

In the sequel, we will assume $N=1$. Now, with the explicit basis diagonalizing $\mathscr{L}_{\beta}$, we are able to write $\mathscr{L}_{\beta, \delta}$ in a Jordan block. More precisely, we recall [24, Lem. 3.1]:

Lemma 2.1 (Jordan block decomposition of $\mathscr{L}_{\beta, \delta}$ ). For all $n \in \mathbb{N}$, there exist two polynomials

$$
\begin{aligned}
& h_{n}=i f_{n}+\sum_{j=0}^{n-1} d_{j, n} f_{j}, \quad \text { where } d_{j, n} \in \mathbb{C}, \\
& \tilde{h}_{n}=(1+i \delta) f_{n}+\sum_{j=0}^{n-1} \tilde{d}_{j, n} f_{j} \quad \text { where } \tilde{d}_{j, n} \in \mathbb{C},
\end{aligned}
$$

of degree $n$ such that

$$
\begin{aligned}
& \mathscr{L}_{\beta, \delta} h_{n}=-\frac{n}{2} h_{n}, \\
& \mathscr{L}_{\beta, \delta} \tilde{h}_{n}=\left(1-\frac{n}{2}\right) \tilde{h}_{n}+c_{n} h_{n-2},
\end{aligned}
$$

with $c_{n}=n(n-1) \beta(1+\delta)^{2}$ (and we take $h_{k} \equiv 0$ for $\left.k<0\right)$. The term of highest order of $h_{n}\left(\right.$ resp. $\left.\tilde{h}_{n}\right)$ is iy $y^{n}$ (resp. $\left.(1+i \delta) y^{n}\right)$.

Proof. See the proof of [24, Lem. 3.1]. For the explicit formulation of $c_{n}$, we look at the imaginary part of order $n-1$ in the equation $\mathscr{L}_{\beta, \delta} \tilde{h}_{n}=\left(1-\frac{n}{2}\right) \tilde{h}_{n}+c_{n} h_{n-2}$.

In addition to this we have the formulas of eigenfunctions $h_{j}, \tilde{h}_{j}, j \in\{1,2, \ldots, 6\}$ in [10]:

Lemma 2.2 (Basis vectors of degree less than or equal to 6). We have

$$
\begin{aligned}
& h_{0}(y)=i, \quad \tilde{h}_{0}=(1+i \delta), \\
& h_{1}(y)=i y, \quad \tilde{h}_{1}=(1+i \delta) y, \\
& h_{2}(y)=i y^{2}+\beta-i(2+\delta \beta), \quad \tilde{h}_{2}=(1+i \delta)\left(y^{2}-2+2 \beta \delta\right), \\
& h_{4}(y)=i y^{4}+y^{2}\left(c_{4,2}+i d_{4,2}\right)+c_{4,0}+i d_{4,0}, \\
& c_{4,2}=6 \beta, \quad d_{4,2}=-6(2+\beta \delta)=-18-6(\beta \delta-1), \\
& c_{4,0}=-4 \beta(3+\beta \delta), \quad d_{4,0}=12-6 \beta^{2}+12 \beta \delta+2 \beta^{2} \delta^{2}, \\
& \tilde{h}_{4}(y)=(1+i \delta) y^{4}+y^{2}\left(12(\beta \delta-1)+i \tilde{d}_{4,2}\right)+\tilde{c}_{4,0}+i \tilde{d}_{4,0}, \\
& \tilde{c}_{4,2}=12(\beta \delta-1), \quad \tilde{d}_{4,2}=0, \\
& \tilde{c}_{4,0}=6 \beta^{2}\left(1+\delta^{2}\right)-12(\beta \delta-1), \quad \tilde{d}_{4,0}=-6 \beta^{2} \delta\left(3 \delta^{2}+7\right)-12 \delta(\beta \delta+1), \\
& h_{6}(y)=i y^{6}+y^{4}\left(c_{6,4}+i d_{6,4}\right)+y^{2}\left(c_{6,2}+i d_{6,2}\right)+c_{6,0}+i d_{6,0}, \\
& c_{6,4}=15 \beta, \quad d_{6,4}=-15(2+\beta \delta),
\end{aligned}
$$




$$
\begin{aligned}
c_{6,2}= & -60 \beta(3+\delta \beta), \quad d_{6,2}=-90 \beta^{2}+180+180 \beta \delta+30 \beta^{2} \delta^{2}, \\
c_{6,0}= & 180 \beta+120 \delta \beta^{2}-45 \beta^{3}+15 \beta^{3} \delta, \\
d_{6,0}= & -180 \beta \delta+55 \delta \beta^{3}-60 \delta^{2} \beta^{2}-5 \beta^{3} \delta^{2}+180 \beta^{2}-120, \\
\tilde{h}_{6}(y)= & (1+i \delta) y^{6}+y^{4}\left(\tilde{c}_{6,4}+i \tilde{d}_{6,4}\right)+y^{2}\left(\tilde{c}_{6,2}+i \tilde{d}_{6,2}\right)+\tilde{c}_{6,0}+i \tilde{d}_{6,0}, \\
\tilde{c}_{6,4}= & 30(\beta \delta-1), \quad \tilde{d}_{6,4}=0, \\
\tilde{c}_{6,2}= & 90 \beta^{2}\left(1+\delta^{2}\right)-180(\beta \delta-1), \\
\tilde{d}_{6,2}= & -90 \beta\left(1+\delta^{2}\right)(3 \beta \delta+4)+180(\beta \delta-1)(\delta-2 \beta), \\
\tilde{c}_{6,0}= & -20 \beta^{2}\left(1+\delta^{2}\right)(11 \beta \delta+21)+120(\beta \delta-1)\left(-2 \beta^{2}+\beta \delta+1\right), \\
\tilde{d}_{6,0}= & 270 \beta\left(1+\delta^{2}\right)(2+\beta \delta)+\beta^{2}\left(1+\delta^{2}\right)\left(140 \beta \delta^{2}-180 \beta \delta+390 \delta\right) \\
& +60(\beta \delta-1)\left(2 \beta^{2} \delta-\beta \delta^{2}+9 \beta-4 \delta\right),
\end{aligned}
$$

Moreover, we have

$$
\begin{aligned}
& \mathscr{L}_{\beta, \delta} \tilde{h}_{0}=\tilde{h}_{0}, \\
& \mathscr{L}_{\beta, \delta} \tilde{h}_{1}=\frac{1}{2} \tilde{h}_{1}, \\
& \mathscr{L}_{\beta, \delta} \tilde{h}_{2}=2 \beta\left(1+\delta^{2}\right) h_{0}=2 i \beta\left(1+\delta^{2}\right), \\
& \mathscr{L}_{\beta, \delta} \tilde{h}_{4}=-\tilde{h}_{4}+12 \beta\left(1+\delta^{2}\right) h_{2}, \\
& \mathscr{L}_{\beta, \delta} \tilde{h}_{6}=-2 \tilde{h}_{6}+30 \beta\left(1+\delta^{2}\right) h_{4} .
\end{aligned}
$$

Corollary 2.1 (Basis for the set of polynomials). The family $\left(h_{n} \tilde{h}_{n}\right)_{n}$ is a basis of $\mathbb{C}[X]$, the $\mathbb{R}$ vector space of complex polynomials.

\subsection{Decomposition of $q$}

For the sake of controlling $q$ in the region $|y|<2 K \sqrt{s}$, we will expand the unknown function $q$ (and not just $\chi q$, where $\chi$ is defined in (16)) with respect to the family $f_{n}$ and with respect to the $h_{n}$. We start by writing

$$
q(y, s)=\sum_{n \leq M} \mathcal{Q}_{n}(s) f_{n}(y)+q_{-}(y, s),
$$

where $f_{n}$ is the eigenfunction of $\mathscr{L}_{\beta}$ defined in $(25), \mathcal{Q}_{n}(s) \in \mathbb{C}, q_{\text {- satisfies }}$

$$
\int q_{-}(y, s) h_{n}(y) \rho(y) d y=0 \quad \text { for all } n \leq M
$$

and $M$ is a fixed even integer satisfying

$$
M \geq 4\left(\sqrt{1+\delta^{2}}+1+2 \max _{i=1,2, y \in \mathbb{R}, s \geq 1}\left|V_{i}(y, s)\right|\right),
$$


with $V_{i=1,2}$ defined in (19). From (29) we have

$$
\mathcal{Q}_{n}(s)=\frac{\int q(y, s) f_{n}(y) \rho_{\beta}(y) d y}{\int f_{n}(y)^{2} \rho_{\beta}(y)} \equiv F_{n}(q(s)),
$$

The function $q_{-}(y, s)$ can be seen as the projection of $q(y, s)$ onto $\left\{f_{j}, j \geq M\right\}$, which corresponds to the eigenvalues smaller than $(1-M) / 2$. We will call it the infinitedimensional part of $q$ and we will denote it $q_{-}=P_{-, M}(q)$. We also introduce $P_{+, M}=$ Id $-P_{-, M}$. Notice that $P_{-, M}$ and $P_{+, M}$ are projections. In the sequel, we will denote $P_{-}=P_{-, M}$ and $P_{+}=P_{+, M}$.

The complementary part $q_{+}=q-q_{-}$will be called the finite-dimensional part of $q$. We will expand it as

$$
q_{+}(y, s)=\sum_{n \leq M} \mathcal{Q}_{n}(s) f_{n}(y)=\sum_{n \leq M} q_{n}(s) h_{n}(y)+\tilde{q}_{n}(s) \tilde{h}_{n}(y),
$$

where $\tilde{q}_{n}, q_{n} \in \mathbb{R}$. Finally, we notice that for all $s$ we have

$$
\int q_{-}(y, s) q_{+}(y, s) \rho_{\beta}(y) d y=0 .
$$

Our purpose is to project (18) in order to write an equation for $q_{n}$ and $\tilde{q}_{n}$. For that we need to write down expressions for $q_{n}$ and $\tilde{q}_{n}$ in terms of $\mathcal{Q}_{n}$. The matrix $\left(h_{n}, \tilde{h}_{n}\right)_{n \leq M}$ in the basis of $\left(i f_{n}, f_{n}\right)$ is upper triangular (see Lemma 2.2). The same holds for its inverse. Thus, we derive from (32),

$$
\begin{aligned}
q_{n} & =\operatorname{Im} \mathcal{Q}_{n}(s)-\delta \operatorname{Re} \mathcal{Q}_{n}(s)+\sum_{j=n+1}^{M} A_{j, n} \operatorname{Im} \mathcal{Q}_{j}(s)+B_{j, n} \operatorname{Re} \mathcal{Q}_{j}(s) \\
& \equiv P_{n, m}(q(s)), \\
\tilde{q}_{n}(s) & =\operatorname{Re} \mathcal{Q}_{n}(s)+\sum_{j=n+1}^{M} \tilde{A}_{j, n} \operatorname{Im} \mathcal{Q}_{j}(s)+\widetilde{B}_{j, n} \operatorname{Re} \mathcal{Q}_{j}(s) \\
& \equiv \widetilde{P}_{n, M}(q(s)),
\end{aligned}
$$

where all the constants are real. Moreover, the coefficients of $\operatorname{Im} \mathcal{Q}_{n}$ and $\operatorname{Re} \mathcal{Q}_{n}$ in the expressions of $q_{n}$ and $\tilde{q}_{n}$ are explicit. This comes from the fact that the same holds for the coefficient of $i f_{n}$ and $f_{n}$ in the expansion of $h_{n}$ and $\tilde{h}_{n}$ (see Lemma 2.1).

Note that the projectors $P_{n, m}(q)$ and $\widetilde{P}_{n, m}(q)$ are well defined thanks to (31). We will project equation (18) on the different modes $h_{n}$ and $\tilde{h}_{n}$. Note from (29) and (32) that

$$
q(y, s)=\left(\sum_{n \leq M} q_{n}(s) h_{n}(y)+\tilde{q}_{n}(s) \tilde{h}_{n}(y)\right)+q_{-}(y, s) .
$$

We should keep in mind that the presentation in (34) is unique. 


\section{Existence}

In this section we prove the existence of a solution $q(s), \theta(s)$ of problem (14)-(44) and further describe the asymptotics of $q$,

$$
\begin{gathered}
q(y, s)=\tilde{h}_{0}(y)\left(\frac{\tilde{\mathscr{A}}_{0}}{s^{2}}\right)+h_{2}(y)\left(\frac{\tilde{\mathcal{A}}_{2}}{s^{2}}\right)+\tilde{h}_{2}(y)\left(\frac{\tilde{\mathcal{A}}_{2}}{s^{2}}+\frac{\widetilde{\mathcal{B}}_{2} \ln s}{s^{2}}\right)+v(y, s), \\
\quad \text { with } \sup _{|y|<M s^{1 / 2}}|v(y, s)| \leq C \frac{\left(1+|y|^{5}\right) \ln ^{2} s}{s^{3}} \text { for all } M>0 \\
\quad \text { and }\left|\theta^{\prime}(s)\right| \leq \frac{C A^{10} \ln ^{2} s}{s^{3}} \text { for all } s \in[-\log T,+\infty),
\end{gathered}
$$

where $\tilde{\mathcal{A}}_{0}, \mathcal{A}_{2}, \tilde{\mathcal{A}}_{2}$ and $\widetilde{\mathcal{B}}_{2}$ are given in Definition 3.1 and $h_{0}(y), h_{2}(y)$ and $\tilde{h}_{2}(y)$ are given in Lemma 2.2.

Hereafter, we denote by $C$ a generic positive constant, depending only on $p, \delta, \beta$ and $K$ introduced in (16), itself depending on $p$.

As a matter of fact, we aim to control the asymptotic (35) by a shrinking set. In fact, we are inspired by the set given in [24] and [10] to introduce a new one that is sharper:

Definition 3.1 (A set shrinking to zero). For all $K>1, A \geq 1$ and $s \geq 1$, we define $\mathcal{V}_{A}(s)$ as the set of all $q \in L^{\infty}(\mathbb{R})$ such that

$$
\begin{array}{l|l}
\left\|q_{e}\right\|_{L^{\infty}(\mathbb{R})} \leq \frac{A^{M+2}}{s^{\frac{1}{2}}}, & \left\|\frac{q-(y)}{1+|y|^{M+1}}\right\|_{L^{\infty}(\mathbb{R})} \leq \frac{A^{M+1}}{s^{\frac{M+2}{2}}} \\
\left|q_{j}\right|,\left|\tilde{q}_{j}\right| \leq \frac{A^{j}}{s^{\frac{j+1}{2}}} \text { for all } 5 \leq j \leq M, \quad\left|q_{0}\right| \leq \frac{1}{s^{2}}, \quad\left|\tilde{q}_{1}\right| \leq \frac{A}{s^{3}}, \quad\left|q_{1}\right| \leq \frac{A^{4}}{s^{3}} .
\end{array}
$$

In addition, the other modes will satisfy the following conditions:

$$
\begin{array}{r}
\left|Q_{4}\right| \leq \frac{A^{7} \ln ^{2} s}{s^{4}} \quad \text { and } \quad\left|\tilde{Q}_{4}\right| \leq \frac{A^{4} \ln ^{2} s}{s^{4}} \\
\left|q_{3}\right| \leq \frac{A^{3}}{s^{4}} \quad \text { and } \quad\left|\tilde{q}_{3}\right| \leq \frac{A^{3}}{s^{4}} \\
\left|Q_{2}\right| \leq \frac{A^{8} \ln ^{2} s}{s^{4}} \quad \text { and } \quad\left|\widetilde{Q}_{2}\right| \leq \frac{A^{10} \ln ^{2} s}{s^{3}}
\end{array}
$$

and

$$
\left|\widetilde{Q}_{0}\right| \leq \frac{A \ln ^{2} s}{s^{4}}
$$

where

$$
\begin{aligned}
Q_{4} & =q_{4}-\left(\frac{1}{2} D_{4,2} \frac{\tilde{q}_{2}}{s}+\left[\frac{C_{4,2} R_{2,1}^{*}}{2}+\frac{R_{4,2}^{*}}{2}\right] \frac{1}{s^{3}}\right), \\
& =q_{4}-\left(\mathcal{A}_{4} \frac{\tilde{q}_{2}}{s}+\frac{\mathcal{B}_{4}}{s^{3}}\right),
\end{aligned}
$$




$$
\begin{aligned}
\tilde{Q}_{4}= & \tilde{q}_{4}-\left(\widetilde{D}_{4,2} \frac{\tilde{q}_{2}}{s}+\frac{1}{s^{3}}\left[\widetilde{C}_{4,2} R_{2,1}^{*}+\widetilde{R}_{4,2}^{*}\right]\right) \\
= & \tilde{q}_{4}-\left(\tilde{\mathscr{A}}_{4} \frac{\tilde{q}_{2}}{s}+\frac{\widetilde{\mathcal{B}}_{4}}{s^{3}}\right), \\
\tilde{Q}_{0}= & \tilde{q}_{0}-\left(\frac{\tilde{q}_{2}}{s}\left[\mu \tilde{L}_{0,2}-\widetilde{D}_{0,2}-\frac{\widetilde{\Theta}_{0,0}^{*} c_{2}}{\kappa}\right]-\frac{\widetilde{R}_{0,1}^{*}}{s^{2}}-\frac{\widetilde{T}_{0,1}^{*} \ln s}{s^{3}}\right) \\
& -\left(\frac{1}{s^{3}}\left[-\tilde{X}_{0}+\mu \widetilde{K}_{0,2} R_{2,1}^{*}-\widetilde{C}_{0,2} \cdot R_{2,1}^{*}-\widetilde{T}_{0,1}^{* *}\right]\right) \\
= & \tilde{q}_{0}-\left(\tilde{\mathcal{A}}_{0} \frac{\tilde{q}_{2}}{s}+\frac{\widetilde{\mathcal{B}}_{0}}{s^{2}}+\frac{\tilde{C}_{0} \ln s}{s^{3}}+\frac{\widetilde{D}_{0}}{s^{3}}\right)
\end{aligned}
$$

and

$$
\begin{aligned}
Q_{2}= & q_{2}-\left(\frac{\tilde{q}_{2}}{s}\left[D_{2,2}-\mu\left(1+\delta^{2}\right)+c_{4} \widetilde{D}_{4,2}+\frac{\Theta_{2,0}^{*} c_{2}}{\kappa}\right]+\frac{R_{2,1}^{*}}{s^{2}}\right) \\
& -\left(\frac{\widetilde{T}_{2,0}^{*} \ln s}{s^{3}}+\frac{1}{s^{3}}\left[X_{2}+c_{4}\left[\widetilde{C}_{4,2} R_{2,1}^{*}+\widetilde{R}_{4,2}^{*}\right]-D_{2,0} \cdot \widetilde{R}_{0,1}^{*}+T_{2,0}^{* *}\right]\right) \\
= & q_{2}-\left(\mathscr{A}_{2} \frac{\tilde{q}_{2}}{s}+\frac{\mathscr{B}_{2}}{s^{2}}+\frac{\mathcal{C}_{2} \ln s}{s^{3}}+\frac{\mathscr{D}_{2}}{s^{3}}\right), \\
\widetilde{Q}_{2}= & \tilde{q}_{2}-\left(\frac{\widetilde{\mathcal{A}}_{2} \ln s}{s^{2}}+\frac{\widetilde{\mathcal{B}}_{2}}{s^{2}}\right)
\end{aligned}
$$

and

$$
\begin{gathered}
\widetilde{\mathcal{A}}_{2}=-\frac{\delta b}{(p-1)^{2}} R_{0,1}^{*}+\left(\mu+\widetilde{C}_{2,2}\right) R_{2,1}^{*}-\widetilde{D}_{2,0} \widetilde{R}_{0,1}^{*}+\widetilde{R}_{2,2}, \\
\begin{cases}\widetilde{\mathscr{B}}_{2}=-\frac{R_{0,1}^{*}-\eta \kappa}{c_{2}}, \text { where } c_{2}=2 \beta\left(1+\delta^{2}\right) & \text { if } \beta \neq 0, \\
\widetilde{\mathscr{B}}_{2} \text { is arbitrary } & \text { if } \beta=0,\end{cases}
\end{gathered}
$$

and

$$
X_{2}=R_{2,2}^{*}+\left(C_{2,2}-\delta \mu\right) R_{2,1}^{*}+\frac{\Theta_{2,0}^{*} R_{0,1}^{*}}{\kappa} \quad \text { and } \quad \widetilde{X}_{0}=\widetilde{R}_{0,2}^{*}-\left(\delta \mu+\widetilde{D}_{0,0}\right) \widetilde{R}_{0,1}^{*} .
$$

Using Definition 3.1, we claim the following:

Claim 3.2 (The size of $\left.q \in \mathcal{V}_{A}\right)$. For all $r \in \mathcal{V}_{A}(s)$ we have the following estimates:

(i) $\|r\|_{L^{\infty}(|y|<2 K \sqrt{s})} \leq C(K) \frac{A^{M+1}}{\sqrt{s}}$ and $\|r\|_{L^{\infty}} \leq C(K) \frac{A^{M+2}}{\sqrt{s}}$.

(ii) For all $y \in \mathbb{R},|r(y)| \leq C \frac{A^{M+1} \ln s}{s^{2}}\left(1+|y|^{M+1}\right)$.

Proof. The proof directly follows from the definition of the shrinking set.

From item (i), our purpose is to control $q$ to stay in $\mathcal{V}(A)$ for $s \geq s_{01}$. Moreover, the bounds in this set help us to conclude the results in the propositions. 
In the following, we aim to choose the initial data.

Definition 3.3 (Choice of initial data). Let us define, for $A \geq 1, s_{0}=-\log T>1$ and $d_{0}, d_{1} \in \mathbb{R}$, the function

$$
\begin{aligned}
\psi_{s_{0}, d_{0}, d_{1}}(y)=[ & \left(\frac{A \ln ^{2} s}{s_{0}^{4}} \tilde{d}_{0}+\frac{\widetilde{\mathcal{B}}_{0}}{s_{0}^{2}}+\frac{\left(\tilde{\mathcal{A}}_{0} \tilde{\mathcal{A}}_{2}+\tilde{\complement}_{0}\right) \ln s_{0}}{s_{0}^{3}}+\frac{\tilde{D}_{0}+\tilde{\mathcal{A}}_{0} \widetilde{\mathcal{B}}_{2}}{s_{0}^{3}}\right) \tilde{h}_{0} \\
& +\frac{A}{s_{0}^{3}} \tilde{d}_{1} \tilde{h}_{1}(y)+d_{0} h_{0}+\left(\frac{\tilde{\mathcal{A}}_{2} \ln s_{0}}{s_{0}^{2}}+\frac{\widetilde{\mathcal{B}}_{2}}{s_{0}^{2}}\right) \tilde{h}_{2} \\
& +\left(\frac{\mathcal{B}_{2}}{s_{0}^{2}}+\frac{\mathcal{D}_{2}+\mathcal{A}_{2} \widetilde{\mathcal{B}}_{2}}{s_{0}^{3}}+\frac{\left(\mathcal{C}_{2}+\mathcal{A}_{2} \tilde{\mathcal{A}}_{2}\right) \ln s_{0}}{s_{0}^{3}}\right) h_{2} \\
& +\left(\frac{\widetilde{\mathcal{B}}_{4}+\tilde{\mathcal{A}}_{4} \tilde{\mathcal{A}}_{2}}{s_{0}^{3}}+\frac{\tilde{\mathcal{A}}_{4} \tilde{\mathcal{A}}_{2} \ln s_{0}}{s_{0}^{3}}\right) \tilde{h}_{4} \\
& \left.+\left(\frac{\mathcal{B}_{4}+\mathcal{A}_{4} \widetilde{\mathcal{B}}_{2}}{s_{0}^{3}}+\frac{\mathcal{A}_{4} \tilde{\mathcal{A}}_{2} \ln s_{0}}{s_{0}^{3}}\right) h_{4}\right] \chi\left(2 y, s_{0}\right),
\end{aligned}
$$

where $s_{0}=-\log T$ and $h_{i}, \tilde{h}_{i}, i=0,1,2,3,4$ are given in Lemma 2.2, $\chi$ is defined by (16) and $d_{0}=d_{0}\left(\tilde{d}_{0}, \tilde{d}_{1}\right)$ will be fixed later in Proposition 3.6 (i). The constants $\tilde{\mathcal{A}}_{i}, \mathcal{A}_{i}$, $\widetilde{\mathcal{B}}_{i}, \mathfrak{B}_{i}, \widetilde{C}_{i}, \mathcal{C}_{i}, \widetilde{\mathfrak{D}}_{i}, \mathscr{D}_{i}$ for $i=0,2,4$ are given by (36)-(40).

Remark 3.4. Let us recall that we will modulate the parameter $\theta$ to kill one of the neutral modes; see equation (44) below. It is natural that this condition must be satisfied for the initial data at $s=s_{0}$. Thus, it is necessary that we choose $d_{0}$ to satisfy condition (44); see (45) below.

So far, in fact, the phase $\theta(s)$ introduced in (11) is arbitrary, as we will show below in Proposition 3.7. We can use a modulation technique to choose $\theta(s)$ in such a way that we impose the condition

$$
P_{0, M}(q(s))=0,
$$

which allows us to kill the neutral direction of the operator $\widetilde{\mathscr{L}}$ defined in (18). Reasonably, our aim is then reduced to the following proposition:

Proposition 3.5 (Existence of a solution trapped in $\mathcal{V}_{A}(s)$ ). There exists $A_{2} \geq 1$ such that for $A \geq A_{2}$ there exists $s_{02}(A)$ such that for all $s_{0} \geq s_{02}(A)$, there exists $\left(\tilde{d}_{0}, \tilde{d}_{1}\right)$ such that if $q$ is the solution of (18)-(44), with initial data given by (43) and (45), then $v \in \mathcal{V}_{A}(s)$ for all $s \geq s_{0}$.

This proposition gives stronger convergence to 0 in $L^{\infty}(\mathbb{R})$.

Let us first be sure that we can choose the initial data such that it starts in $\mathcal{V}_{A}\left(s_{0}\right)$. In other words, we will define a set where we will select the good parameters $\left(\tilde{d}_{0}, \tilde{d}_{1}\right)$ that will give the conclusion of Proposition 3.5. More precisely, we have the following result:

Proposition 3.6 (Properties of initial data). For each $A \geq 1$, there exists $s_{03}(A)>1$ such that for all $s_{0} \geq s_{03}$, we have the following properties: 
(i) $P_{0, M}\left(i \chi\left(2 y, s_{0}\right)\right) \neq 0$ and the parameter $d_{0}\left(s_{0}, \tilde{d}_{0}, \tilde{d}_{1}\right)$ given by

$$
\begin{aligned}
& d_{0}\left(s_{0}, \tilde{d}_{0}, \tilde{d}_{1}\right)=-\frac{A}{s_{0}^{3}} \tilde{d}_{1} \frac{P_{0, M}\left(\tilde{h}_{1} \chi\left(2 y, s_{0}\right)\right)}{P_{0, M}\left(i \chi\left(2 y, s_{0}\right)\right)} \\
& -\left(\frac{A \ln ^{2} s_{0}}{s_{0}^{4}} \tilde{d}_{0}+\frac{\widetilde{\mathcal{B}}_{0}}{s_{0}^{2}}+\frac{\left(\tilde{\mathcal{A}}_{0} \tilde{\mathscr{A}}_{2}+\tilde{C}_{0}\right) \ln s_{0}}{s_{0}^{3}}+\frac{\widetilde{D}_{0}+\tilde{\mathcal{A}}_{0} \widetilde{\mathscr{B}}_{2}}{s_{0}^{3}}\right) \\
& \times \frac{P_{0, M}\left(\tilde{h}_{0} \chi\left(2 y, s_{0}\right)\right)}{P_{0, M}\left(i \chi\left(2 y, s_{0}\right)\right)} \\
& -\left(\frac{\tilde{\mathcal{A}}_{2} \ln s_{0}}{s_{0}^{2}}+\frac{\widetilde{\mathcal{B}}_{2}}{s_{0}^{2}}\right) \frac{P_{0, M}\left(\tilde{h}_{2} \chi\left(2 y, s_{0}\right)\right)}{P_{0, M}\left(i \chi\left(2 y, s_{0}\right)\right)} \\
& -\left(\frac{\mathscr{B}_{2}}{s_{0}^{2}}+\frac{\mathscr{D}_{2}+\mathcal{A}_{2} \widetilde{\mathcal{B}}_{2}}{s_{0}^{3}}+\frac{\left(\mathcal{C}_{2}+\mathcal{A}_{2} \tilde{\mathcal{A}}_{2}\right) \ln s_{0}}{s_{0}^{3}}\right) \frac{P_{0, M}\left(h_{2} \chi\left(2 y, s_{0}\right)\right)}{P_{0, M}\left(i \chi\left(2 y, s_{0}\right)\right)} \\
& -\left(\frac{\widetilde{\mathscr{B}}_{4}+\tilde{\mathcal{A}}_{4} \tilde{\mathcal{A}}_{2}}{s_{0}^{3}}+\frac{\tilde{\mathcal{A}}_{4} \tilde{\mathcal{A}}_{2} \ln s_{0}}{s_{0}^{3}}\right) \frac{P_{0, M}\left(\tilde{h}_{4} \chi\left(2 y, s_{0}\right)\right)}{P_{0, M}\left(i \chi\left(2 y, s_{0}\right)\right)} \\
& -\left(\frac{\mathscr{B}_{4}+\mathcal{A}_{4} \widetilde{\mathcal{B}}_{2}}{s_{0}^{3}}+\frac{\mathcal{A}_{4} \tilde{\mathcal{A}}_{2} \ln s_{0}}{s_{0}^{3}}\right) \frac{P_{0, M}\left(h_{4} \chi\left(2 y, s_{0}\right)\right)}{P_{0, M}\left(i \chi\left(2 y, s_{0}\right)\right)}
\end{aligned}
$$

is well defined, where $\chi$ is defined in (16) and the constants $\tilde{\mathcal{A}}_{i}, \mathcal{A}_{i}, \widetilde{\mathcal{B}}_{i}, \mathscr{B}_{i}, \tilde{\complement}_{i}, \boldsymbol{C}_{i}$ for $i=0,2,4$ are given by (36)-(40).

(ii) If $\psi$ is given by (43) and (45) with $d_{0}$ defined by (45) then there exists a quadrilateral $\mathcal{D}_{s_{0}} \subset[-2,2]^{2}$ such that the mapping

$$
\left(\tilde{d}_{0}, \tilde{d}_{1}\right) \rightarrow\left(\tilde{\Psi}_{0}=\tilde{\psi}_{0}-\left(\frac{\widetilde{\mathcal{B}}_{0}}{s_{0}^{2}}+\frac{\left(\tilde{\mathscr{A}}_{0} \tilde{\mathscr{A}}_{2}+\tilde{\mathscr{C}}_{0}\right) \ln s_{0}}{s_{0}^{3}}+\frac{\tilde{\mathscr{D}}_{0}+\tilde{\mathscr{A}}_{0} \widetilde{\mathcal{B}}_{2}}{s_{0}^{3}}\right), \widetilde{\psi}_{1}\right)
$$

(where $\psi$ stands for $\left.\psi_{s_{0}, \tilde{d}_{0}, \tilde{d}_{1}}\right)$ is linear, one to one from $\mathscr{D}_{s_{0}}$ onto $\left[-\frac{A \ln ^{2} s_{0}}{s_{0}^{4}}, \frac{A \ln ^{2} s_{0}}{s_{0}^{4}}\right] \times$ $\left[-\frac{A}{s_{0}^{3}}, \frac{A}{s_{0}^{3}}\right]$. Moreover, it is of degree 1 on the boundary.

(iii) For all $\left(\tilde{d}_{0}, \tilde{d}_{1}\right) \in \mathscr{D}_{s_{0}}$ we have

$$
\psi_{e} \equiv 0, \quad \psi_{0}=0
$$

for all $3 \leq i \leq M, i \neq 4,1 \leq j \leq M, j \neq\{2,4\}$ and for some $\gamma>0$,

$$
\left|\widetilde{\psi}_{i}\right|+\left|\psi_{j}\right| \leq C e^{-\gamma s_{0}}
$$

and

$$
\left|\widetilde{\Psi}_{i}\right|+\left|\Psi_{j}\right| \leq C e^{-\gamma s_{0}} \quad \text { for } i, j=\{2,4\},
$$

where $\widetilde{\Psi}_{i}$ and $\Psi_{i}$ are defined as in (36)-(40).

Moreover, it holds that $\left\|\frac{\psi_{-}(y)}{\left(1+|y|^{M+1}\right)}\right\|_{L^{\infty}(\mathbb{R})} \leq C A / s_{0}^{\frac{M}{4}+1}$. 
(iv) For all $\left(\tilde{d}_{0}, \tilde{d}_{1}\right) \in \mathscr{D}_{s_{0}}, \psi_{s_{0}, \tilde{d}_{0}, \tilde{d}_{1}} \in \mathcal{V}_{A}\left(s_{0}\right)$ with strict inequalities except for $\left(\tilde{\psi}_{0}, \tilde{\psi}_{1}\right)$. Proof. The proof is the same as [24, Prop. 4.2] and [10, Prop. 4.5].

In the following, we find a local-in-time solution for equation (18) coupled with condition (44).

Proposition 3.7 (Local-in-time solution and modulation for problem (18)-(44) with initial data (43)-(45)). For all $A \geq 1$, there exists $T_{3}(A) \in(0,1 / e)$ such that for all $T \leq T_{3}$, the following holds: for all $\left(\tilde{d}_{0}, \tilde{d}_{1}\right) \in D_{T}$, there exists $s_{\max }>s_{0}=-\log T$ such that problem (18)-(44) with initial data at $s=s_{0}$,

$$
\left(q\left(s_{0}\right), \theta\left(s_{0}\right)\right)=\left(\psi_{s_{0}, \tilde{d}_{0}, \tilde{d}_{1}}, 0\right),
$$

where $\psi_{s_{0}, \tilde{d}_{0}, \tilde{d}_{1}}$ is given by (43) and (45), has a unique solution $q(s), \theta(s)$ satisfying $q(s) \in$ $V_{A+1}(s)$ for all $s \in\left[s_{0}, s_{\max }\right)$.

Proof. The proof is quite similar to [24, Prop. 4.4] and [10, Prop. 4.6].

Let us now give the proof of Proposition 3.5.

Proof of Proposition 3.5. Let us consider $A \geq 1, s_{0} \geq s_{03},\left(\tilde{d}_{0}, \tilde{d}_{1}\right) \in \mathscr{D}_{s_{0}}$, where $s_{03}$ is given by Proposition 3.6. From the existence theory (which follows from the Cauchy problem for equation (CGL)), starting in $\mathcal{V}_{A}\left(s_{0}\right)$ which is in $\mathcal{V}_{A+1}\left(s_{0}\right)$, the solution stays in $\mathcal{V}_{A}(s)$ until some maximal time $s_{*}=s_{*}\left(\tilde{d}_{0}, \tilde{d}_{1}\right)$. Then either

- $s_{*}\left(\tilde{d}_{0}, \tilde{d}_{1}\right)=\infty$ for some $\left(\tilde{d}_{0}, \tilde{d}_{1}\right) \in \mathscr{D}_{s_{0}}$ and the proof is complete;

- $s_{*}\left(\tilde{d}_{0}, \tilde{d}_{1}\right)<\infty$ for any $\left(\tilde{d}_{0}, \tilde{d}_{1}\right) \in \mathcal{D}_{s_{0}}$ and we argue by contradiction. By continuity and the definition of $s_{*}$, the solution on $s_{*}$ is in the boundary of $\mathcal{V}_{A}\left(s_{*}\right)$. Then, by definition of $\mathcal{V}_{A}\left(s_{*}\right)$, at least one of the inequalities in that definition is an equality. Owing to the following proposition, this can happen only for the first two components $\tilde{q}_{0}, \tilde{q}_{1}$.

Precisely we have the following result:

Proposition 3.8 (Control of $q(s)$ by $\left(q_{0}(s), q_{1}(s)\right)$ in $\mathcal{V}_{A}(s)$ ). There exists $A_{4} \geq 1$ such that for each $A \geq A_{4}$, there exists $s_{04} \in \mathbb{R}$ such that for all $s_{0} \geq s_{04}$. The following holds:

If $q$ is a solution of (18) with initial data at $s=s_{0}$ given by (43) and (45) with $\left(\tilde{d}_{0}, \tilde{d}_{1}\right) \in \mathcal{D}_{s_{0}}$, and $q(s) \in \mathcal{V}(A)(s)$ for all $s \in\left[s_{0}, s_{1}\right]$, with $q\left(s_{1}\right) \in \partial \mathcal{V}_{A}\left(s_{1}\right)$ for some $s_{1} \geq s_{0}$, then we have the following properties:

(i) (Smallness of the modulation parameter $\theta$ defined in (11)) For all $s \in\left[s_{0}, s_{1}\right]$,

$$
\left|\theta^{\prime}(s)\right| \leq \frac{C A^{10} \ln ^{2} s}{s^{3}} .
$$

(ii) (Reduction to a finite-dimensional problem) We have

$$
\left(\tilde{Q}_{0}\left(s_{1}\right), \tilde{q}_{1}\left(s_{1}\right)\right) \in \partial\left(\left[-\frac{A \ln ^{2} s_{1}}{s_{1}^{4}}, \frac{A \ln ^{2} s_{1}}{s_{1}^{4}}\right] \times\left[-\frac{A}{s_{1}^{3}}, \frac{A}{s_{1}^{3}}\right]\right) .
$$


(iii) (Transverse crossing) There exists $\omega \in\{-1,1\}$ such that

$$
\begin{gathered}
\omega \widetilde{Q}_{0}\left(s_{1}\right)=\frac{A}{s_{1}^{4}} \quad \text { and } \quad \omega \frac{d \widetilde{Q}_{0}\left(s_{1}\right)}{d s}\left(s_{1}\right)>0, \\
\omega \tilde{q}_{1}\left(s_{1}\right)=\frac{A}{s_{1}^{3}} \quad \text { and } \quad \omega \frac{d \tilde{q}_{1}}{d s}\left(s_{1}\right)>0 .
\end{gathered}
$$

Proof. See the proof in Section 3.1.

Assume the result of the previous proposition, for which the proof is given below in Section 3.1, and continue the proof of Proposition 3.5. Let $A \geq A_{4}$ and $s_{0} \geq s_{04}(A)$. It follows from Proposition 3.8(ii) that $\left(\widetilde{Q}_{0}, \tilde{q}_{1}\left(s_{*}\right)\right) \in \partial\left(\left[-\frac{A}{s_{1}^{4}}, \frac{A}{s_{1}^{4}}\right] \times\left[-\frac{A}{s_{1}^{3}}, \frac{A}{s_{1}^{3}}\right]\right)$, and the function

$$
\begin{aligned}
\phi: \mathscr{D}_{s_{0}} & \rightarrow \partial\left([-1,1]^{2}\right) \\
\left(\tilde{d}_{0}, \tilde{d}_{1}\right) & \rightarrow\left(\frac{s_{*}^{4}}{A \ln ^{2} s^{*}} \tilde{Q}_{0}, \frac{s_{*}^{3}}{A} \tilde{q}_{1}\right)_{\left(\tilde{d}_{0}, \tilde{d}_{1}\right)}\left(s_{*}\right), \quad \text { with } s_{*}=s_{*}\left(\tilde{d}_{0}, \tilde{d}_{1}\right)
\end{aligned}
$$

is well defined. Then it follows from Proposition 3.8 (iii) that $\phi$ is continuous. On the other hand, using Proposition 3.6 (ii)-(iv) together with the fact that $q\left(s_{0}\right)=\psi_{s_{0}, \tilde{d}_{0}, \tilde{d}_{1}}$, we see that when $\left(\tilde{d}_{0}, \tilde{d}_{1}\right)$ is in the boundary of the rectangle $\mathscr{D}_{s_{0}}$ we have strict inequalities for the other components.

Applying the transverse crossing property given by Proposition 3.8 (iii), we see that $q(s)$ leaves $\mathcal{V}_{A}(s)$ at $s=s_{0}$, hence $s_{*}\left(\tilde{d}_{0}, \tilde{d}_{1}\right)=s_{0}$. Using Proposition 3.6 (ii), we see that the restriction of $\phi$ to the boundary is of degree 1. A contradiction then follows from the index theory. Thus there exists a value $\left(\tilde{d}_{0}, \tilde{d}_{1}\right) \in \mathscr{D}_{s_{0}}$ such that for all $s \geq s_{0}, q_{s_{0}, d_{0}, d_{1}}(s) \in$ $\mathcal{V}_{A}(s)$. This concludes the proof of Proposition 3.5.

Using Proposition 3.8 (i), we get the bound on $\theta^{\prime}(s)$. This concludes the proof of (35).

\subsection{Reduction to a finite-dimensional problem}

In the following we give the proof of Proposition 3.8.

The idea of the proof is to project equation (18) on the different components of the decomposition (34). More precisely, we claim that Proposition 3.8 is a consequence of the following proposition:

Proposition 3.9. There exists $A_{5} \geq 1$ such that for all $A \geq A_{5}$, there exists $s_{5}(A)$ such that the following holds for all $s_{0} \geq s_{5}$ : assuming that for all $s \in\left[\tau, s_{1}\right]$ for some $s_{1} \geq \tau \geq s_{0}$, $q(s) \in \mathcal{V}_{A}(s)$ and $q_{0}(s)=0$, then the following holds for all $s \in\left[\tau, s_{1}\right]$ :

(i) (Smallness of the modulation parameter)

$$
\left|\theta^{\prime}(s)\right| \leq \frac{C A^{10} \ln ^{2} s}{s^{3}} .
$$


(ii) (ODE satisfied by the expanding mode) For $m=0$ and 1 we have

$$
\left|\widetilde{Q}_{0}^{\prime}(s)-Q_{0}(s)\right| \leq \frac{C \ln ^{2} s}{s^{4}}
$$

and

$$
\left|\tilde{q}_{1}^{\prime}-\frac{1}{2} \tilde{q}_{1}\right| \leq \frac{C}{s^{3}}
$$

(iii) (ODE satisfied by the null mode)

$$
\left|\tilde{Q}_{2}^{\prime}(s)-\frac{2 \widetilde{Q}_{2}}{s}\right| \leq \frac{C A^{8} \ln ^{2} s}{s^{3}} .
$$

(iv) (Control of negative modes)

$$
\begin{aligned}
& \left|q_{1}(s)\right| \leq e^{-\frac{(s-\tau)}{2}}\left|q_{1}(\tau)\right|+\frac{C A^{3}}{s^{3}}, \\
& \left|Q_{2}(s)\right| \leq e^{-(s-\tau)}\left|Q_{2}(\tau)\right|+\frac{C A^{7} \ln ^{2} s}{s^{4}}, \\
& \left|q_{3}\right| \leq e^{-\frac{3}{2}(s-\tau)}\left|q_{3}(\tau)\right|+\frac{C A^{2}}{s^{4}}, \\
& \left|\tilde{q}_{3}\right| \leq e^{-\frac{s-\tau}{2}}\left|\tilde{q}_{3}(\tau)\right|+\frac{C A^{2}}{s^{4}}, \\
& \left|Q_{4}(s)\right| \leq e^{-2(s-\tau)}\left|Q_{4}(\tau)\right|+\frac{C A^{6} \ln ^{2} s}{s^{4}}, \\
& \left|\widetilde{Q}_{4}(s)\right| \leq e^{-(s-\tau)}\left|\widetilde{Q}_{4}(\tau)\right|+\frac{C A^{3} \ln ^{2} s}{s^{4}}, \\
& \left|q_{j}(s)\right| \leq e^{-j \frac{(s-\tau)}{2}}\left|q_{j}(\tau)\right|+\frac{C A^{j-1}}{s^{\frac{j+1}{2}}} \text { for all } 5 \leq j \leq M, \\
& \left|\tilde{q}_{j}(s)\right| \leq e^{-(j-2) \frac{(s-\tau)}{2}}\left|\tilde{q}_{j}(\tau)\right|+\frac{C A^{j-1}}{s^{\frac{j+1}{2}}} \text { for all } 5 \leq j \leq M, \\
& \left\|\frac{q_{-}(y, s)}{1+|y|^{M+1}}\right\|_{L^{\infty}} \leq e^{-\frac{M+1}{4}(s-\tau)}\left\|\frac{q_{-}(\tau)}{1+|y|^{M+1}}\right\|_{L^{\infty}}+C \frac{A^{M}}{s^{\frac{M+2}{2}}}, \\
& \left\|q_{e}(y, s)\right\|_{L^{\infty}} \leq e^{-\frac{(s-\tau)}{2(p-1)}}\left\|q_{e}(\tau)\right\|_{L^{\infty}}+\frac{C A^{M+1}}{\sqrt{\tau}}(1+s-\tau),
\end{aligned}
$$

where $\widetilde{Q}_{0}, Q_{2}, \widetilde{Q}_{2}, Q_{4}$ and $\widetilde{Q}_{4}$ are defined by (36)-(40).

Proof. Briefly speaking, the main idea of the proof of Proposition 3.9 is to project equations (14) and (18) according to the decomposition (34). Due to the lengthy proof with many technical computations, we will give the complete proof in Section 3.2.

Proof of Proposition 3.8. Let us now focus on the proof of Proposition 3.8 assuming Proposition 3.9 holds. Indeed, we will take $A_{4} \geq A_{5}$. Hence, we can use the conclusion of Proposition 3.9. 
(i) The proof follows from (i) of Proposition 3.9. Indeed, by choosing $T_{4}$ small enough, we can make $s_{0}=-\log T$ bigger than $s_{5}(A)$.

(ii) We notice that from Claim 3.2 and the fact that $q_{0}(s)=0$, it is enough to prove that for all $s \in\left[s_{0}, s_{1}\right]$,

$$
\begin{aligned}
& \left|\widetilde{Q}_{2}(s)\right|=\left|\tilde{q}_{2}(s)-\left(\frac{\widetilde{\mathscr{A}}_{2} \ln s}{s^{2}}+\frac{\widetilde{\mathcal{B}}_{2}}{s^{2}}\right)\right|<\frac{A^{10} \ln ^{2} s}{s^{3}}, \\
& \left\|q_{e}\right\|_{L^{\infty}(\mathbb{R})} \leq \frac{A^{M+2}}{2 s^{\frac{1}{2}}}, \quad \quad\left\|\frac{q-(y, s)}{1+|y|^{M+1}}\right\|_{L^{\infty}} \leq \frac{A^{M+1}}{2 s^{\frac{M+2}{2}}}, \\
& \left|q_{j}\right|,\left|\tilde{q}_{j}\right| \leq \frac{A^{j}}{2 s^{\frac{j+1}{2}}} \quad \text { for all } 5 \leq j \leq M, \quad\left|q_{1}\right| \leq \frac{A^{4}}{2 s^{3}}, \\
& \left|Q_{2}\right| \leq \frac{A^{8} \ln ^{2} s}{2 s^{4}} \\
& \left|Q_{4}\right|,\left|\widetilde{Q}_{4}\right| \leq \frac{A^{7} \ln s}{2 s^{4}} .
\end{aligned}
$$

In fact, the estimates in (47) are similar to [10, Prop. 4.7]. For that reason, we only focus on the proof of (46). Indeed, we will use a contradictory argument: we assume that there exists $s_{*} \in\left[s_{0}, s_{1}\right]$ such that

$$
\widetilde{Q}_{2}\left(s_{*}\right)=\left(\tilde{q}_{2}\left(s_{*}\right)-\left(\frac{\tilde{\mathscr{A}}_{2} \ln s_{*}}{s_{*}^{2}}+\frac{\widetilde{\mathcal{B}}_{2}}{s_{*}^{2}}\right)\right)=\omega \frac{A^{10} \ln ^{2} s_{*}}{s_{*}^{3}}
$$

for all

$$
s \in\left[s_{0}, s_{*}[\right.
$$

and

$$
\left|\tilde{q}_{2}(s)-\left(\frac{\tilde{\mathscr{A}}_{2} \ln s}{s^{2}}+\frac{\tilde{\mathcal{B}}_{2}}{s^{2}}\right)\right|<\frac{A^{10} \ln ^{2} s}{s^{3}},
$$

where $\omega= \pm 1$. As a matter of fact, we can reduce to the positive case where $\omega=1$ (the case $\omega=-1$ also works in the same way). Note by Proposition 3.6 (iv) that

$$
\left|\tilde{q}_{2}\left(s_{0}\right)-\left(\frac{\tilde{\mathcal{A}}_{2} \ln s_{0}}{s_{0}^{2}}+\frac{\widetilde{\mathcal{B}}_{2}}{s_{0}^{2}}\right)\right|<\frac{A^{10} \ln ^{2} s_{0}}{s_{0}^{3}},
$$

thus $s_{*}>s_{0}$, and the interval $\left[s_{0}, s_{*}\right]$ is not empty.

Using the continuity of $\widetilde{Q}_{2}$ and the definition of $s_{*}$, it is clear that $\widetilde{Q}_{2}\left(s_{*}\right)$ is the maximal value of $\widetilde{Q}_{2}$ in $\left[s_{*}-\varepsilon, s_{*}\right]$ with $\varepsilon>0$ and small enough. Then, recalling from Proposition 3.9 (iii) that

$$
\left|\widetilde{Q}_{2}^{\prime}+2 \frac{\widetilde{Q}_{2}}{s}\right| \leq \frac{C A^{8} \ln ^{2} s}{s^{4}}
$$

hence it follows that

$$
\tilde{Q}_{2}^{\prime}\left(s_{*}\right) \leq-\frac{\tilde{Q}_{2}}{s}+\frac{C A^{8} \ln ^{2} s}{s^{4}} \leq \frac{\left(-2 A^{10}+C A^{8}\right) \ln ^{2} s}{s^{4}}<0,
$$


provided that $A$ is large enough. Then $\widetilde{Q}_{2}$ has to decrease in $\left[s_{*}-\varepsilon_{1}, s_{*}\right]$ which implies a contradiction with the assumption that $\widetilde{Q}_{2}$ admits a maximum at $s_{*}$. In other words, (46) holds. Finally, the proof of Proposition 3.8 is concluded.

\subsection{Proof of Proposition 3.9}

In this section we focus on the proof of Proposition 3.9. The idea is mainly based on the technique in [24], [28] and [10]. In fact, it involves the projection equations (14) and (18) to get equations satisfied by the different coordinates of the decomposition (34). Let us summarize the proof:

- In the first part of Section 3.2.1 we deal with equation (18) to write equations satisfied by $\tilde{q}_{j}$ and $q_{j}$. Then we prove (i), (ii), (iii) and (iv) (except the two last identities) of Proposition 3.9.

- In the second part of Section 3.2.1 we first derive from equation (18) an equation satisfied by $q_{-}$and prove the last but one identity in Proposition 3.9 (iv).

- In Section 3.2.2 we project equation (14) (which is simpler than (18)) to write an equation satisfied by $q_{e}$ and prove the last identity in Proposition 3.9 (iv).

3.2.1. The finite-dimensional part: $\boldsymbol{q}_{+}$- We now divide the proof into two steps:

- In part 1 we give the details of projections of equation (18) to get ODEs, satisfied by modes $\tilde{q}_{j}$ and $q_{j}$.

- In part 2 we prove Proposition 3.9 (i), (ii), (iii), together with the estimates concerning $\tilde{q}_{j}$ and $q_{j}$ in (iv).

Part 1: The projection of equation (18) on the eigenfunction of the operator $\mathscr{L}_{\beta, \delta}$. In the following, we will find the main contribution in the projections $\widetilde{P}_{n, M}$ and $P_{n, M}$ of the six terms appearing in equation (18): $\partial_{s} q, \mathscr{L}_{\beta, \delta} q,-i\left(\frac{v}{2 \sqrt{s}}+\frac{\mu}{s}+\theta^{\prime}(s)\right) q, V_{1} q+V_{2} \bar{q}$, $B(q, y, s)$ and $R^{*}\left(\theta^{\prime}, y, s\right)$.

First term: $\frac{\partial q}{\partial s}$. From (33), we directly derive

$$
\widetilde{P}_{n, M}\left(\frac{\partial q}{\partial s}\right)=\tilde{q}_{n}^{\prime} \quad \text { and } \quad P_{n, M}\left(\frac{\partial q}{\partial s}\right)=q_{n}^{\prime} .
$$

Second term: $\mathscr{L}_{\beta, \delta} q$, where $\mathscr{L}_{\beta, \delta}$ is defined as in (19). We will use the following lemma from [24]:

Lemma 3.10 (Projection of $\mathscr{L}_{\beta, \delta}$ on $\tilde{h}_{n}$ and $h_{n}$ for $n \leq M$ ).

(a) If $n \leq M-2$, then

$$
\left|P_{n, M}\left(\mathscr{L}_{\beta, \delta} q\right)-\left(-\frac{n}{2} q_{n}(s)+c_{n+2} \tilde{q}_{n+2}\right)\right| \leq C\left\|\frac{q_{-}}{1+|y|^{M+1}}\right\|_{L^{\infty}},
$$

where $c_{n}$ is given in Lemma 2.1. Moreover, we have the following: if $M-1 \leq n \leq M$, then

$$
\left|P_{n, M}\left(\mathscr{L}_{\beta, \delta} q\right)+\frac{n}{2} q_{n}(s)\right| \leq C\left\|\frac{q_{-}}{1+|y|^{M+1}}\right\|_{L^{\infty}} .
$$


(b) If $n \leq M$, then the projection of $\mathscr{L}_{\beta, \delta}$ on $\tilde{h}_{n}$ satisfies

$$
\left|\widetilde{P}_{n, M}\left(\mathscr{L}_{\beta, \delta} q\right)-\left(1-\frac{n}{2}\right) \tilde{q}_{n}(s)\right| \leq C\left\|\frac{q-}{1+|y|^{M+1}}\right\|_{L^{\infty}} .
$$

Proof. The proof is similar to the proof of [24, Lem. 5.1].

Using Lemma 3.10 and the fact that $q(s) \in \mathcal{V}_{A}(s)$ (see Definition 3.1) we can improve the error by the following:

Corollary 3.1. For all $A \geq 1$, there exists $s_{9} \geq 1$ such that for all $s \geq s_{9}(A)$, if $q(s) \in$ $\mathcal{V}_{A}(s)$, then we have the following properties:

(a) For $n=0$ we have

$$
\left|P_{0, M}\left(\mathscr{L}_{\beta, \delta} q\right)-c_{2} \tilde{q}_{2}\right| \leq C \frac{A^{M+1}}{s^{\frac{M+2}{2}}} .
$$

(b) For $1 \leq n \leq M-1$ we have

$$
\left|P_{n, M}\left(\mathscr{L}_{\beta, \delta} q\right)+\frac{n}{2} q_{n}(s)\right| \leq C \frac{A^{n+2}}{s^{\frac{n+3}{2}}} .
$$

In particular, we have a smaller bound for $P_{2, M}\left(\mathscr{L}_{\beta, \delta} q\right)$ :

$$
\left|P_{2, M}\left(\mathscr{L}_{\beta, \delta} q\right)+q_{2}-c_{4} \tilde{q}_{4}\right| \leq \frac{A^{M+1}}{s^{\frac{M+2}{2}}} .
$$

(c) For $n=M$ we have

$$
\left|P_{M, M}\left(\mathscr{L}_{\beta, \delta} q\right)+\frac{M}{2} q_{M}(s)\right| \leq C \frac{A^{M+1}}{s^{\frac{M+2}{2}}} .
$$

(d) For $0 \leq n \leq M$ we have

$$
\left|\widetilde{P}_{n, M}\left(\mathscr{L}_{\beta, \delta} q\right)-\left(1-\frac{n}{2}\right) \tilde{q}_{n}(s)\right| \leq C \frac{A^{M+1}}{s^{\frac{M+1}{2}}} .
$$

Third term: $-i\left(\frac{\mu}{s}-\eta \frac{\ln s}{s^{2}}+\frac{\eta}{s^{2}}+\theta^{\prime}(s)\right) q$. It is enough to project $i q$, from (33); we recall [24, Lem. 5.3]:

Lemma 3.11 (Projection of the term $-i\left(\frac{\mu}{s}-\eta \frac{\ln s}{s^{2}}+\frac{\eta}{s^{2}}+\theta^{\prime}(s)\right) q$ on $h_{n}$ and $\tilde{h}_{n}$ for $n \leq M)$. We have the following equalities:

(i) the projection on $h_{n}$,

$$
\begin{aligned}
P_{n, M} & \left(-i\left(\frac{\mu}{s}-\eta \frac{\ln s}{s^{2}}+\frac{\eta}{s^{2}}+\theta^{\prime}(s)\right) q\right) \\
& =-\left(\frac{\mu}{s}-\eta \frac{\ln s}{s^{2}}+\frac{\eta}{s^{2}}+\theta^{\prime}(s)\right)\left(\delta q_{n}+\left(1+\delta^{2}\right) \tilde{q}_{n}+\sum_{j=n+1}^{M} K_{n, j} q_{j}+L_{n, j} \tilde{q}_{j}\right),
\end{aligned}
$$


where $K_{n, j}$ and $L_{n, j}$ are defined by

$$
\begin{aligned}
K_{n, j} & =P_{n, M}\left(i h_{j}\right), \\
L_{n, j} & =P_{n, M}\left(i \tilde{h}_{j}\right)
\end{aligned}
$$

(ii) the projection on $\tilde{h}_{n}$,

$$
\begin{aligned}
& \widetilde{P}_{n, M}\left(-i\left(\frac{\mu}{s}-\eta \frac{\ln s}{s^{2}}+\frac{\eta}{s^{2}}+\theta^{\prime}(s)\right) q\right) \\
& \quad=-\left(\frac{\mu}{s}-\eta \frac{\ln s}{s^{2}}+\frac{\eta}{s^{2}}+\theta^{\prime}(s)\right)\left(-q_{n}-\delta \tilde{q}_{n}+\sum_{j=n+1}^{M} \tilde{K}_{n, j} q_{j}+\tilde{L}_{n, j} \tilde{q}_{j}\right),
\end{aligned}
$$

where $\tilde{K}_{n, j}$ and $\tilde{L}_{n, j}$ are defined by

$$
\begin{aligned}
\tilde{K}_{n, j} & =\widetilde{P}_{n, M}\left(i h_{j}\right), \\
\tilde{L}_{n, j} & =\widetilde{P}_{n, M}\left(i \tilde{h}_{j}\right) .
\end{aligned}
$$

Using the fact that $q(s) \in \mathcal{V}_{A}(s)$ is defined in Definition 3.1, the error estimates can be improved:

Corollary 3.2. For all $A \geq 1$, there exists $s_{10}(A) \geq 1$ such that for all $s \geq s_{10}(A)$, if $q \in \mathcal{V}_{A}(s)$ and $\left|\theta^{\prime}(s)\right| \leq \frac{C \bar{A}^{10}}{s^{5 / 2}}$, then we have the following properties:

(a) For all $1 \leq n \leq M$ we have

$$
\left|P_{n, M}\left(-i\left(\frac{\mu}{s}-\eta \frac{\ln s}{s^{2}}+\frac{\eta}{s^{2}}+\theta^{\prime}(s)\right) q\right)\right| \leq C \frac{A^{n}}{s^{\frac{n+3}{2}}} .
$$

(b) For $1 \leq n \leq M$ we have

$$
\left|\widetilde{P}_{n, M}\left(-i\left(\frac{\mu}{s}-\eta \frac{\ln s}{s^{2}}+\frac{\eta}{s^{2}}+\theta^{\prime}(s)\right) q\right)\right| \leq C \frac{A^{n}}{s^{\frac{n+3}{2}}} .
$$

In particular, when $n=0,2,4$, we can get smaller bounds as follows:

(c) For $n=0$ we have the following in particular:

$$
\begin{aligned}
\mid P_{0, M}( & \left.-i\left(\frac{\mu}{s}-\eta \frac{\ln s}{s^{2}}+\frac{\eta}{s^{2}}+\theta^{\prime}(s)\right) q\right) \\
& +\left(\frac{\mu}{s}-\eta \frac{\ln s}{s^{2}}+\frac{\eta}{s^{2}}+\theta^{\prime}(s)\right)\left\{\delta q_{0}+\left(1+\delta^{2}\right) \tilde{q}_{0}+K_{0,2} q_{2}+L_{0,2} \tilde{q}_{2}\right\} \mid \\
\leq & C \frac{A^{4} \ln s}{s^{4}} \\
\mid \widetilde{P}_{0, M}( & \left.-i\left(\frac{\mu}{s}-\eta \frac{\ln s}{s^{2}}+\frac{\eta}{s^{2}}+\theta^{\prime}(s)\right) q\right) \\
& +\left(\frac{\mu}{s}-\eta \frac{\ln s}{s^{2}}+\frac{\eta}{s^{2}}+\theta^{\prime}(s)\right)\left\{-q_{0}-\delta \tilde{q}_{0}+\tilde{K}_{0,2} q_{2}+\tilde{L}_{0,2} \tilde{q}_{2}\right\} \mid \\
\leq & C \frac{A^{4} \ln s}{s^{4}} .
\end{aligned}
$$


(d) For $n=2$ we have

$$
\begin{aligned}
\mid P_{2, M}( & \left.-i\left(\frac{\mu}{s}-\eta \frac{\ln s}{s^{2}}+\frac{\eta}{s^{2}}+\theta^{\prime}(s)\right) q\right) \\
& +\left(\frac{\mu}{s}-\eta \frac{\ln s}{s^{2}}+\frac{\eta}{s^{2}}+\theta^{\prime}(s)\right)\left[\delta q_{2}+\left(1+\delta^{2}\right) \tilde{q}_{2}\right] \mid \\
\leq & C \frac{A^{4} \ln s}{s^{4}} \\
\mid \widetilde{P}_{2, M}( & \left.-i\left(\frac{\mu}{s}-\eta \frac{\ln s}{s^{2}}+\frac{\eta}{s^{2}}+\theta^{\prime}(s)\right) q\right) \\
& +\left(\frac{\mu}{s}-\eta \frac{\ln s}{s^{2}}+\frac{\eta}{s^{2}}+\theta^{\prime}(s)\right)\left(-q_{2}-\delta \tilde{q}_{2}+\tilde{K}_{2,4} q_{4}+\tilde{L}_{2,4} \tilde{q}_{4}\right) \mid \\
\leq & C \frac{A^{5}}{s^{4}} .
\end{aligned}
$$

(e) For $n=3$ we have

$$
\begin{aligned}
& \left|P_{3, M}\left(-i\left(\frac{\mu}{s}-\eta \frac{\ln s}{s^{2}}+\frac{\eta}{s^{2}}+\theta^{\prime}(s)\right) q\right)\right| \leq C \frac{A^{2}}{s^{4}}, \\
& \left|\widetilde{P}_{3, M}\left(-i\left(\frac{\mu}{s}-\eta \frac{\ln s}{s^{2}}+\frac{\eta}{s^{2}}+\theta^{\prime}(s)\right) q\right)\right| \leq C \frac{A^{2}}{s^{4}} .
\end{aligned}
$$

(f) For $n=4$ we have

$$
\begin{aligned}
& \left|P_{4, M}\left(-i\left(\frac{\mu}{s}-\eta \frac{\ln s}{s^{2}}+\frac{\eta}{s^{2}}+\theta^{\prime}(s)\right) q\right)\right| \leq C \frac{A^{5}}{s^{4}}, \\
& \left|\widetilde{P}_{4, M}\left(-i\left(\frac{\mu}{s}-\eta \frac{\ln s}{s^{2}}+\frac{\eta}{s^{2}}+\theta^{\prime}(s)\right) q\right)\right| \leq C \frac{A^{5}}{s^{4}} .
\end{aligned}
$$

Fourth term: $V_{1} q+V_{2} \bar{q}$. We recall [24, Lem. 5.5]:

Lemma 3.12 (Projections of $V_{1} q$ and $V_{2} \bar{q}$ ).

(i) It holds that

$$
\left|V_{i}(y, s)\right| \leq C \frac{\left(1+|y|^{2}\right)}{s} \quad \text { for all } y \in \mathbb{R} \text { and } s \geq 1,
$$

and for all $k \in \mathbb{N}^{*}$,

$$
V_{i}(y, s)=\sum_{j=1}^{k} \frac{1}{s^{j}} W_{i, j}(y)+\widetilde{W}_{i, k}(y, s),
$$

where $W_{i, j}$ is an even polynomial of degree $2 j$ and $\tilde{W}_{i, k}(y, s)$ satisfies

$$
\text { for all } s \geq 1 \text { and }|y| \leq \sqrt{s}, \quad\left|\tilde{W}_{i, k}(y, s)\right| \leq C \frac{\left(1+|y|^{2 k+2}\right)}{s^{k+1}} .
$$


(ii) The projections of $V_{1} q$ and $V_{2} \bar{q}$ on $h_{n}$ and $\tilde{h}_{n}$ satisfy

$$
\begin{aligned}
& \left|\widetilde{P}_{n, M}\left(V_{1} q\right)\right|+\left|P_{n, M}\left(V_{1} q\right)\right| \\
& \quad \leq \frac{C}{s} \sum_{j=n-2}^{M}\left(\left|\tilde{q}_{j}\right|+\left|\hat{q}_{j}\right|\right)+\sum_{j=0}^{n-3} \frac{C}{s^{\frac{n-j}{2}}}\left(\left|\tilde{q}_{j}\right|+\left|\hat{q}_{j}\right|\right)+\frac{C}{s}\left\|\frac{q_{-}}{1+|y|^{M+1}}\right\|_{L^{\infty}},
\end{aligned}
$$

and the same holds for $V_{2} \bar{q}$.

Remark 3.13. Note that, when $n \leq 2$, the first sum in (57) runs for $j=0$ to $M$ and the second sum does not exist.

By the fact that $q(s) \in \mathcal{V}_{A}(s)$, the error estimates can be bounded improved as follows: Corollary 3.3. For all $A \geq 1$, there exists $s_{11}(A) \geq 1$ such that for all $s \geq s_{11}(A)$, if $q \in \mathcal{V}_{A}(s)$, then for $3 \leq n \leq M$ we have

$$
\left|\widetilde{P}_{n}\left(V_{1} q+V_{2} \bar{q}\right)\right|+\left|P_{n}\left(V_{1} q+V_{2} \bar{q}\right)\right| \leq \frac{C A^{n-2}}{s^{\frac{n+1}{2}}} .
$$

Now we study the asymptotics of $\widetilde{P}_{2, M}\left(V_{1} q\right), \widetilde{P}_{2, M}\left(V_{2} \bar{q}\right), P_{0, M}\left(V_{1} q\right)$ and $P_{0, M}\left(V_{2} \bar{q}\right)$ :

Lemma 3.14. Using the definitions of $V_{1}, V_{2}$, the following hold:

(i) It holds that for $i=1,2$,

$$
\text { for all } s \geq 1 \text { and }|y| \leq s^{1 / 2}, \quad\left|V_{i}(y, s)-\frac{1}{s} W_{i, 1}(y)\right| \leq \frac{C}{s^{2}}\left(1+|y|^{4}\right),
$$

where

$$
\begin{aligned}
& W_{1,1}(y)=-\frac{(p+1) b}{2(p-1)^{2}}(1+i \delta)\left(y^{2}-2(1-\delta \beta)\right) \\
& W_{2,1}(y)=-(1+i \delta) \frac{b}{2(p-1)^{2}}(p-1+2 i \delta)\left(y^{2}-2(1-\beta \delta)\right) .
\end{aligned}
$$

(ii) The projections of $V_{1} q$ and $V_{2} \bar{q}$ on $\hat{h}_{n}$ and $\tilde{h}_{n}$ satisfy

$$
\begin{gathered}
\left|\widetilde{P}_{n, M}\left(V_{1} q+V_{2} \bar{q}\right)-\frac{1}{s} \sum_{j \geq 0}\left[\widetilde{C}_{n, j} q_{j}+\widetilde{D}_{n, j} \tilde{q}_{j}\right]\right| \\
\leq \frac{C}{s^{2}} \sum_{j \geq 0}\left[\left|\hat{q}_{j}\right|+\left|\tilde{q}_{j}\right|\right]+\frac{1}{s}\left\|\frac{q-(., s)}{1+|y|^{M}}\right\|_{L^{\infty}},
\end{gathered}
$$

and

$$
\begin{gathered}
\left|P_{n, M}\left(V_{1} q+V_{2} \bar{q}\right)-\frac{1}{s} \sum_{j \geq 0}\left[C_{n, j} q_{j}+D_{2, j} \tilde{q}_{j}\right]\right| \\
\leq \frac{C}{s^{2}} \sum_{j \geq 0}\left[\left|\hat{q}_{j}\right|+\left|\tilde{q}_{j}\right|\right]+\frac{1}{s}\left\|\frac{q_{-}(., s)}{1+|y|^{M}}\right\|_{L^{\infty}},
\end{gathered}
$$


where for all $n, j \geq 0$ we have

$$
\begin{aligned}
& C_{n, j}=P_{n, M}\left(W_{1,1} h_{j}+W_{2,1} \bar{h}_{j}\right) \widetilde{C}_{n, j}=\widetilde{P}_{n, M}\left(W_{1,1} h_{j}+W_{2,1} \bar{h}_{j}\right), \\
& D_{n, j}=P_{n, M}\left(W_{1,1} \tilde{h}_{j}+W_{2,1} \overline{\tilde{h}}_{j}\right) \tilde{D}_{n, j}=\widetilde{P}_{n, M}\left(W_{1,1} \tilde{h}_{j}+W_{2,1} \overline{\tilde{h}}_{j}\right) .
\end{aligned}
$$

In particular, using the fact that $q(s) \in \mathcal{V}_{A}(s)$, the error estimates can be improved as follows:

Corollary 3.4. For all $A \geq 1$, there exists $s_{12}(A) \geq 1$ such that for all $s \geq s_{12}(A)$, if $q(s) \in \mathcal{V}_{A}(s)$, then

$$
\begin{aligned}
& \left|P_{0, M}\left(V_{1} q+V_{2} \bar{q}\right)-\left(C_{0,0} \frac{q_{0}}{s}+D_{0,0} \frac{\tilde{q}_{0}}{s}+C_{0,2} \frac{q_{2}}{s}+D_{0,2} \frac{\tilde{q}_{2}}{s}\right)\right| \leq C \frac{\ln s}{s^{4}}, \\
& \left|\widetilde{P}_{0, M}\left(V_{1} q+V_{2} \bar{q}\right)-\left(\widetilde{D}_{0,0} \frac{\tilde{q}_{0}}{s}+\widetilde{C}_{0,2} \frac{q_{2}}{s}+\widetilde{D}_{0,2} \frac{\tilde{q}_{2}}{s}\right)\right| \leq C \frac{\ln s}{s^{4}}, \\
& \left|P_{2, M}\left(V_{1} q+V_{2} \bar{q}\right)-\left(\frac{D_{2,0} \tilde{q}_{0}}{s}+\frac{C_{2,2} q_{2}}{s}+\frac{D_{2,2} \tilde{q}_{2}}{s}\right)\right| \leq C \frac{\ln s}{s^{4}}, \\
& \left|\widetilde{P}_{2, M}\left(V_{1} q+V_{2} \bar{q}\right)-\frac{1}{s}\left\{\tilde{q}_{0} \widetilde{D}_{2,0}+q_{2} \widetilde{C}_{2,2}+\tilde{q}_{2} \widetilde{D}_{2,2}\right\}\right| \leq C \frac{\ln s}{s^{4}}, \\
& \left|P_{4, M}\left(V_{1} q+V_{2} \bar{q}\right)-\left(C_{4,2} \frac{q_{2}}{s}+D_{4,2} \frac{\tilde{q}_{2}}{s}\right)\right| \leq C \frac{\ln s}{s^{4}}, \\
& \left|\widetilde{P}_{4, M}\left(V_{1} q+V_{2} \bar{q}\right)-\left(\widetilde{C}_{4,2} \frac{q_{2}}{s}+\widetilde{D}_{4,2} \frac{\tilde{q}_{2}}{s}\right)\right| \leq C \frac{\ln s}{s^{4}}
\end{aligned}
$$

and

$$
\begin{aligned}
& \left|P_{3, M}\left(V_{1} q+V_{2} \bar{q}\right)\right| \leq \frac{C A^{2}}{s^{4}}, \\
& \left|\widetilde{P}_{3, M}\left(V_{1} q+V_{2} \bar{q}\right)\right| \leq \frac{C A^{2}}{s^{4}} .
\end{aligned}
$$

Fifth term: $B(q, y, s)$.

$$
\begin{aligned}
B(q, y, s)= & (1+i \delta) \\
& \times\left(|\varphi+q|^{p-1}(\varphi+q)-|\varphi|^{p-1} \varphi-|\varphi|^{p-1} q-\frac{p-1}{2}|\varphi|^{p-3} \varphi(\varphi \bar{q}+\bar{\varphi} q)\right) .
\end{aligned}
$$

We have the following lemma:

Lemma 3.15. The function $B=B(q, y, s)$ can be decomposed for all $s \geq 1$ and $|q| \leq 1$ as

$$
\begin{aligned}
& \sup _{|y| \leq s^{1 / 2}}\left|B-\sum_{l=0}^{M} \sum_{\substack{0 \leq j, k \leq M+1 \\
2 \leq j+k \leq M+1}} \frac{1}{s^{l}}\left[B_{j, k}^{l}\left(\frac{y}{s^{1 / 2}}\right) q^{j} \bar{q}^{k}+\widetilde{B}_{j, k}^{l}(y, s) q^{j} \bar{q}^{k}\right]\right| \\
& \quad \leq C|q|^{M+2}+\frac{C}{s^{\frac{M+1}{2}}},
\end{aligned}
$$


where $B_{j, k}^{l}\left(\frac{y}{s^{1 / 2}}\right)$ is an even polynomial of degree less than or equal to $M$ and the rest of $\widetilde{B}_{j, k}^{l}(y, s)$ satisfies

$$
\text { for all } s \geq 1 \text { and }|y|<s^{1 / 2}, \quad\left|\widetilde{B}_{j, k}^{l}(y, s)\right| \leq C \frac{1+|y|^{M+1}}{s^{\frac{M+1}{2}}} .
$$

Moreover,

$$
\text { for all } s \geq 1 \text { and }|y|<s^{1 / 2}, \quad\left|B_{j, k}^{l}\left(\frac{y}{s^{1 / 2}}\right)+\widetilde{B}_{j, k}^{l}(y, s)\right| \leq C .
$$

On the other hand, in the region $|y| \geq s^{1 / 2}$ we have

$$
|B(q, y, s)| \leq C|q|^{\bar{p}}
$$

for some constant $C$ where $\bar{p}=\min (p, 2)$.

Proof. See [24, proof of Lem. 5.9, p. 1646].

Lemma 3.16 (The quadratic term $B(q, y, s)$ ). For all $A \geq 1$, there exists $s_{13} \geq 1$ such that for all $s \geq s_{13}$, if $q(s) \in \mathcal{V}_{A}(s)$, then

(a) the projections of $B(q, y, s)$ on $h_{n}$ and on $\tilde{h}_{n}$, for $n \geq 3$ satisfy

$$
\left|\widetilde{P}_{n, M}(B(q, y, s))\right|+\left|P_{n, M}(B(q, y, s))\right| \leq C \frac{A^{n}}{s^{\frac{n+2}{2}}} ;
$$

(b) for $n=0,1,2,3,4$ we have

$$
\left|\widetilde{P}_{n, M}(B(q, y, s))\right|+\left|P_{n, M}(B(q, y, s))\right| \leq \frac{C \ln ^{2} s}{s^{4}} .
$$

Proof. See [24, Lem. 5.10].

Sixth term: $R^{*}\left(\theta^{\prime}, y, s\right)$. In the following, we expand $R^{*}$ as a power series of $\frac{1}{s}$ as $s \rightarrow$ $\infty$, uniformly for $|y| \leq s^{1 / 2}$.

Lemma 3.17 (Power series of $R^{*}$ as $s \rightarrow \infty$ ). For all $n \in \mathbb{N}$,

$$
R^{*}\left(\theta^{\prime}, y, s\right)=\Pi_{n}\left(\theta^{\prime}, y, s\right)+\widetilde{\Pi}_{n}\left(\theta^{\prime}, y, s\right),
$$

where

$$
\begin{aligned}
\Pi_{n}\left(\theta^{\prime}, y, s\right)= & \sum_{k=0}^{n-1} \frac{1}{s^{k+1}} P_{k}(y) \\
& -i\left(-\eta \frac{\ln s}{s^{2}}+\frac{\eta}{s^{2}}+\theta^{\prime}(s)\right)\left(\frac{a}{s}(1+i \delta)+\sum_{k=0}^{n-1} e_{k} \frac{y^{2 k}}{s^{k}}\right),
\end{aligned}
$$

and

$$
\text { for all }|y|<s^{1 / 2}, \quad\left|\widetilde{\Pi}_{n}\left(\theta^{\prime}, y, s\right)\right| \leq C\left(1+s\left|\theta^{\prime}(s)\right|\right) \frac{\left(1+|y|^{2 n}\right)}{s^{n+1}},
$$

where $P_{k}$ is a polynomial of order $2 k$ for all $k \geq 1$ and $e_{k} \in \mathbb{R}$. 
In particular,

$$
\begin{gathered}
\sup _{|y| \leq s^{1 / 2} \mid} \mid R^{*}\left(\theta^{\prime}, y, s\right)-\sum_{k=0}^{1} \frac{1}{s^{k+1}} P_{k}(y)+i\left(-\eta \frac{\ln s}{s^{2}}+\frac{\eta}{s^{2}}+\theta^{\prime}\right) \\
\quad \times\left[\kappa+\frac{(1+i \delta)}{s}\left(a-\frac{b \kappa y^{2}}{(p-1)^{2}}\right)\right] \mid \\
\leq C\left(\frac{1+|y|^{4}}{s^{3}}+C\left(\frac{\ln s}{s^{2}}+\left|\theta^{\prime}\right|\right) \frac{y^{4}}{s^{2}}\right) .
\end{gathered}
$$

Proof. The proof is similar to [24, Lem. 5.11].

In the following, we introduce $F_{j}\left(R^{*}\right)(\theta, s)$ as the projection of the rest term $R^{*}\left(\theta^{\prime}, y, s\right)$ on the standard Hermite polynomial, introduced in Lemma 2.1.

Lemma 3.18 (Projection of $R^{*}$ on the eigenfunction of $\mathscr{L}$ ). It holds that $F_{j}\left(R^{*}\right)\left(\theta^{\prime}, s\right) \equiv$ 0 when $j$ is odd, and $\left|F_{j}\left(R^{*}\right)\left(\theta^{\prime}, s\right)\right| \leq C \frac{1+s\left|\theta^{\prime}(s)\right|}{s^{j / 2+1}}$ when $j$ is even and $j \geq 4$.

Proof. See [24, Lem. 5.12].

More precisely, we can describe the projection of $R^{*}$ as follows:

Lemma 3.19 (Projection of $R^{*}$ on the eigenfunctions $\tilde{h}$ and $h_{n}$ ). Let us consider $R^{*}$ defined as in the above, then the following hold:

(i) For $j \geq 4$ which is even, then $\widetilde{P}_{j}\left(R^{*}\right)\left(\theta^{\prime}, s\right)$ and $P_{j}\left(R^{*}\right)\left(\theta^{\prime}, s\right)$ are $O\left(\frac{1+s\left|\theta^{\prime}\right|}{s^{j / 2+1}}\right)$.

(ii) For all $j$ odd we have $\widetilde{P}_{j}\left(R^{*}\right)\left(\theta^{\prime}, s\right)=P_{j}\left(R^{*}\right)\left(\theta^{\prime}, s\right)=0$.

(iii) For $j=0$ we have

$$
\begin{aligned}
P_{0, M}\left(R^{*}\left(\theta^{\prime}(s), s\right)\right)= & \frac{R_{0,0}^{*}}{s}+\frac{R_{0,1}^{*}}{s^{2}}+\frac{R_{0,2}^{*}}{s^{3}}+\theta^{\prime}(s)\left(-\kappa+\frac{\Theta_{0,0}^{*}}{s}+O\left(\frac{1}{s^{2}}\right)\right) \\
& +\frac{\ln s}{s^{2}}\left(\eta \kappa+\frac{T_{0,0}^{*}}{s}+O\left(\frac{1}{s^{2}}\right)\right)+\frac{1}{s^{2}}\left(-\eta \kappa+\frac{T_{0,0}^{* *}}{s}+O\left(\frac{1}{s^{2}}\right)\right) \\
& +O\left(\frac{1}{s^{4}}\right), \\
\widetilde{P}_{0, M}\left(R^{*}\left(\theta^{\prime}(s), s\right)\right)= & \frac{\widetilde{R}_{0,0}^{*}}{s}+\frac{\widetilde{R}_{0,1}^{*}}{s^{2}}+\frac{\widetilde{R}_{0,2}^{*}}{s^{3}}+\theta^{\prime}(s)\left(\frac{\widetilde{\Theta}_{0,0}^{*}}{s}+O\left(\frac{1}{s^{2}}\right)\right) \\
& +\frac{\ln s}{s^{2}}\left(\frac{\widetilde{T}_{0,0}^{*}}{s}+O\left(\frac{1}{s^{2}}\right)\right)+\frac{1}{s^{2}}\left(\frac{\widetilde{T}_{0,0}^{* *}}{s}+O\left(\frac{1}{s^{2}}\right)\right)+O\left(\frac{1}{s^{4}}\right) .
\end{aligned}
$$

(iv) For $j=2$ we have

$$
\begin{aligned}
P_{2, M}\left(R^{*}\left(\theta^{\prime}(s), s\right)\right)= & \frac{R_{2,1}^{*}}{s}+\frac{R_{2,2}^{*}}{s^{3}}+\theta^{\prime}(s)\left(\frac{\Theta_{2,0}^{*}}{s}+O\left(\frac{1}{s^{2}}\right)\right) \\
& +\frac{\ln s}{s^{2}}\left(\frac{T_{2,0}^{*}}{s}+O\left(\frac{1}{s^{2}}\right)\right)+\frac{1}{s^{2}}\left(\frac{T_{2,0}^{* *}}{s}+O\left(\frac{1}{s^{2}}\right)\right)+O\left(\frac{1}{s^{4}}\right),
\end{aligned}
$$




$$
\begin{aligned}
\widetilde{P}_{2, M}\left(R^{*}\left(\theta^{\prime}(s), s\right)\right)= & \frac{\widetilde{R}_{2,1}^{*}}{s^{2}}+\frac{\widetilde{R}_{2,1}^{*}}{s^{3}}+\theta^{\prime}(s)\left(\frac{\widetilde{\Theta}_{2,0}^{*}}{s}+O\left(\frac{1}{s^{2}}\right)\right) \\
& +\frac{\ln s}{s^{2}}\left(\frac{\widetilde{T}_{2,0}^{*}}{s}+O\left(\frac{1}{s^{2}}\right)\right)+\frac{1}{s^{2}}\left(\frac{\widetilde{T}_{2,0}^{* *}}{s}+O\left(\frac{1}{s^{2}}\right)\right)+O\left(\frac{1}{s^{4}}\right),
\end{aligned}
$$

where $R_{j, k}^{*}, \widetilde{R}_{j, k}^{*}, \Theta_{j, k}^{*}, \widetilde{\Theta}_{j, k}^{*}$ are constants, depending on $p, \delta, \beta$ only. For more details see Appendix $\mathrm{C}$ and equation (92).

(v) In particular, we choose

$$
\left\{\begin{array}{l}
a=\frac{2(1-\delta \beta) b}{(p-1)^{2}} \\
\mu=-\frac{2 \beta b}{(p-1)^{2}}\left(1+\delta^{2}\right) \\
b=\frac{(p-1)^{2}}{4\left(p-\delta^{2}-(p+1) \delta \beta\right)}
\end{array}\right.
$$

Then we have

$$
R_{0,0}^{*}=\widetilde{R}_{0,0}^{*}=\widetilde{R}_{2,1}^{*}=0
$$

Proof. For the details, we kindly refer readers to Appendix C.

Part 2: Proof of Proposition 3.9. In this part, we consider $A \geq 1$ and take $s$ large enough that Part 1 is satisfied.

- Proof of item (i): We control $\theta^{\prime}(s)$. From the projection of (18) on $h_{0}(y)=i$, we obtain

$$
\begin{aligned}
q_{0}^{\prime}= & c_{2} \tilde{q}_{2}+P_{0, M}\left(-i\left(\frac{\mu}{s}-\frac{\eta \ln s}{s^{2}}+\frac{\eta}{s^{2}}+\theta^{\prime}\right) q\right) \\
& +P_{0, M}\left(V_{1} q+V_{2} \bar{q}\right)+P_{0, M}(B)+P_{0, M}\left(R^{*}\left(\theta^{\prime}(s), s\right)\right),
\end{aligned}
$$

where $c_{2}=2 \beta\left(1+\delta^{2}\right)$, as defined in Lemma 2.1. In addition to this, from the fact that $q_{0} \equiv 0$ by the modulation, we also obtain

$$
q_{0}^{\prime} \equiv 0
$$

Using the fact that $q \in \mathcal{V}_{A}(s)$, given in Definition 3.1, together with Corollaries 3.1 and 3.4, Lemmas 3.10, 3.16 and 3.19, we obtain the following:

$$
\begin{aligned}
& P_{0, M}\left(\mathscr{L}_{\delta, \beta} q\right)=c_{2} \tilde{q}_{2}+O\left(\frac{1}{s^{\frac{M+2}{2}}}\right) \\
& P_{0, M}\left(-i\left(\frac{\mu}{s}-\frac{\eta \log s}{s^{2}}+\frac{\eta}{s^{2}}+\theta^{\prime}\right) q\right) \\
& =-\left(\frac{\mu}{s}-\frac{\eta \log s}{s^{2}}+\frac{\eta}{s^{2}}+\theta^{\prime}(s)\right)\left\{\delta q_{0}+\left(1+\delta^{2}\right) \tilde{q}_{0}+K_{0,2} q_{2}+L_{0,2} \tilde{q}_{2}\right\}+O\left(\frac{\ln s}{s^{4}}\right)
\end{aligned}
$$

and

$$
P_{0, M}\left(V_{1} q+V_{2} \bar{q}\right)=C_{0,0} \frac{q_{0}}{s}+D_{0,0} \frac{\tilde{q}_{0}}{s}+C_{0,2} \frac{q_{2}}{s}+D_{0,2} \frac{\tilde{q}_{2}}{s}+O\left(\frac{\ln s}{s^{4}}\right),
$$




$$
\begin{aligned}
P_{0, M}(B(q))= & O\left(\frac{\ln ^{2} s}{s^{4}}\right), \\
P_{0, M}\left(R^{*}\right)= & \left\{-\kappa+\frac{\Theta_{0,0}^{*}}{s}+O\left(\frac{1}{s^{2}}\right)\right\} \theta^{\prime}(s)+\frac{\left(R_{0,1}^{*}-\eta \kappa\right)}{s^{2}}+\frac{R_{0,2}^{*}}{s^{3}}+\frac{\eta \kappa \ln s}{s^{2}} \\
& +T_{0,1}^{*} \frac{\ln s}{s^{3}}+O\left(\frac{\ln s}{s^{4}}\right), \\
\mid & \kappa \theta^{\prime}(s)+c_{2} \tilde{q}_{2}-\frac{\mu}{s}\left\{\left(1+\delta^{2}\right) \tilde{q}_{0}+K_{0,2} q_{2}+L_{0,2} \tilde{q}_{2}\right\}+D_{0,0} \frac{\tilde{q}_{0}}{s} \\
+ & C_{0,2} \frac{q_{2}}{s}+D_{0,2} \frac{\tilde{q}_{2}}{s}+(\eta \kappa) \frac{\ln s}{s^{2}}+\frac{\left(R_{0,1}^{*}-\eta \kappa\right)}{s^{2}}+\frac{\left(R_{0,2}^{*}-T_{0,0}^{* *}\right)}{s^{3}}+T_{0,0}^{*} \frac{\ln s}{s^{3}} \\
+ & \frac{c_{2} \Theta_{0,0}^{*}}{\kappa} \frac{\tilde{q}_{2}}{s}+\frac{\Theta_{0,0}^{*}\left(R_{0,1}^{*}-\eta \kappa\right)}{\kappa} \frac{1}{s^{3}}+\frac{\Theta_{0,0}^{*}(\eta \kappa) \ln s}{\kappa s^{3}} \mid \leq C \frac{\ln ^{2} s}{s^{4}} .
\end{aligned}
$$

In particular, we use again the fact that $q \in \mathcal{V}_{A}$,

$$
\left|c_{2} \tilde{q}_{2}(s)+\frac{\left(R_{0,1}^{*}-\eta \kappa\right)}{s^{2}}+\frac{\eta \kappa \ln s}{s^{2}}\right| \leq \frac{A^{10} \ln ^{2} s}{s^{3}},
$$

which can be written

$$
\left|\tilde{q}_{2}(s)-\frac{\tilde{\mathcal{A}}_{2} \ln s}{s^{2}}-\frac{\widetilde{\mathcal{B}}_{2}}{s^{2}}\right| \leq \frac{A^{10} \ln ^{2} s}{s^{3}},
$$

where

$$
\tilde{\mathcal{A}}_{2}=-\frac{\eta \kappa}{c_{2}} \quad \text { and } \quad \widetilde{\mathcal{B}}_{2}=-\frac{\left(R_{0,1}^{*}-\eta \kappa\right)}{c_{2}} .
$$

Thus, we obtain

$$
\left|\theta^{\prime}(s)\right| \leq \frac{C A^{10} \ln ^{2} s}{s^{3}}
$$

and

$$
\left|-\kappa \theta^{\prime}(s)+c_{2} \tilde{q}_{2}(s)+\frac{\left(R_{0,1}^{*}-\eta \kappa\right)}{s^{2}}+\frac{\eta \kappa \ln s}{s^{2}}\right| \leq \frac{C \ln s}{s^{3}},
$$

which concludes Proposition 3.9 (i).

- Proof of item (iii): Let us project equation (18) on $\tilde{h}_{2}$. We get

$$
\begin{aligned}
\tilde{q}_{2}^{\prime}= & \widetilde{P}_{2, M}\left(\mathscr{L}_{\beta, \delta} q\right)+\widetilde{P}_{2, M}\left(-i\left(\frac{\mu}{s}-\frac{\eta \ln s}{s^{2}}+\frac{\eta}{s^{2}}+\theta^{\prime}(s)\right) q\right)+\widetilde{P}_{2, M}\left(V_{1} q+V_{2} \bar{q}\right) \\
& +\widetilde{P}_{2, M}(B(q))+\widetilde{P}_{2, M}\left(R^{*}\left(\theta^{\prime}(s), s\right)\right) .
\end{aligned}
$$

We repeat the same process as for $\tilde{q}_{0}$. Using the fact that $q(s) \in \mathcal{V}_{A}(s)$ for all $s \in\left[\tau, s_{1}\right]$, by Corollaries 3.1 and 3.4, and Lemmas 3.10, 3.16, 3.19, we obtain the following bounds for the terms on the right-hand side of (77):

$$
\begin{aligned}
& \widetilde{P}_{2, M}\left(\partial_{s} q\right)=\partial_{s} \tilde{q}_{2}, \\
& \left|\widetilde{P}_{2, M}\left(\mathscr{L}_{\beta, \delta} q\right)\right| \leq \frac{A^{M+1}}{s^{\frac{M+2}{2}}} .
\end{aligned}
$$

In particular, we also have the following expansion: 
Terms coming from $\widetilde{P}_{2, M}\left(-i\left(\frac{\mu}{s}-\frac{\eta \ln s}{s^{2}}+\frac{\eta}{s^{2}}+\theta^{\prime}(s)\right) q\right)$. We have

$$
\widetilde{P}_{2, M}\left(-i\left(\frac{\mu}{s}-\frac{\eta \ln s}{s^{2}}+\frac{\eta}{s^{2}}+\theta^{\prime}(s)\right) q\right)=-\frac{\mu}{s}\left(-q_{2}-\delta \tilde{q}_{2}\right)+O\left(\frac{\ln s}{s^{4}}\right) .
$$

Terms coming from $\widetilde{P}_{2, M}\left(V_{1} q+V_{2} \bar{q}\right)$. We have

$$
\widetilde{P}_{2, M}\left(V_{1} q+V_{2} \bar{q}\right)=\frac{1}{s}\left\{\tilde{q}_{0} \widetilde{D}_{2,0}+q_{2} \widetilde{C}_{2,2}+\tilde{q}_{2} \widetilde{D}_{2,2}\right\}+O\left(\frac{\ln s}{s^{4}}\right) .
$$

This yields

$$
\tilde{P}_{2}\left(V_{1} q+V_{2} \bar{q}\right)=\frac{\widetilde{D}_{2,2}}{s} \tilde{q}_{2}+\frac{1}{s^{3}}\left\{\widetilde{C}_{2,2} R_{2,1}^{*}-\widetilde{D}_{2,0} \widetilde{R}_{0,1}^{*}\right\}+O\left(\frac{\ln s}{s^{4}}\right) .
$$

Terms coming from $\widetilde{\boldsymbol{P}}_{2, \boldsymbol{M}}(\boldsymbol{B}(\boldsymbol{q}))$. We have

$$
\left|\widetilde{P}_{2, M}(B)\right| \leq \frac{C \ln ^{2} s}{s^{4}} .
$$

Terms coming from $\widetilde{\boldsymbol{P}}_{\mathbf{2}, \boldsymbol{M}}\left(\boldsymbol{R}^{*}\right)$. We have

$$
\begin{aligned}
\widetilde{P}_{2, M}\left(R^{*}\right)= & \widetilde{T}_{2,0}^{*} \frac{\ln s}{s^{3}}+\frac{\left(\widetilde{R}_{2,2}^{*}+\widetilde{T}_{2,0}^{* *}\right)}{s^{3}}+\frac{\theta^{\prime}(s) \kappa}{s} \frac{-\delta b}{(p-1)^{2}}+O\left(\frac{\ln s}{s^{4}}\right) \\
= & \widetilde{T}_{2,0}^{*} \frac{\ln s}{s^{3}}+\frac{\left(\widetilde{R}_{2,2}^{*}+\widetilde{T}_{2,0}^{* *}\right)}{s^{3}}+\left(c_{2} \tilde{q}_{2}(s)+\frac{\left(R_{0,1}^{*}-\eta \kappa\right)}{s^{2}}+\frac{\eta \kappa \ln s}{s^{2}}\right) \frac{-\delta b}{s(p-1)^{2}} \\
& +O\left(\frac{\ln s}{s^{4}}\right) \\
= & -\frac{c_{2} \delta b}{(p-1)^{2} s} \tilde{q}_{2}-\frac{\delta b}{(p-1)^{2} s^{3}} R_{0,1}^{*}+\frac{\widetilde{R}_{2,2}^{*}}{s^{3}}+O\left(\frac{\ln s}{s^{4}}\right) .
\end{aligned}
$$

Note that we combined the facts given in (75) and (76), and $\mu, b$ and $a$ are as given in (71). Finally, by adding these estimates, we obtain

$$
\begin{aligned}
\tilde{q}_{2}^{\prime}= & \frac{\tilde{q}_{2}}{s}\left\{\delta \mu+\widetilde{D}_{2,2}-\frac{c_{2} \delta b}{(p-1)^{2}}\right\} \\
& +\frac{1}{s^{3}}\left\{\mu R_{2,1}^{*}+\widetilde{C}_{2,2} R_{2,1}^{*}-\widetilde{D}_{2,0} \widetilde{R}_{0,1}^{*}+\widetilde{R}_{2,2}-\frac{\delta b}{(p-1)^{2}} R_{0,1}^{*}\right\} \\
& +O\left(\frac{\ln ^{2} s}{s^{4}}\right) .
\end{aligned}
$$

Let us remark that even though there exists the order $\frac{\ln s}{s^{3}}$ in the ODE of $\tilde{q}_{2}$, it will be canceled when we add all terms in the ODE. From the explicit formulas of $\mu, b, c_{2}$ and $\widetilde{D}_{2,2}$, we can compute

$$
\delta \mu+\widetilde{D}_{2,2}-\frac{c_{2} \delta b}{(p-1)^{2}}=-2
$$


In addition to this, using the definition of $\widetilde{Q}_{2}$ given as in (40), we establish that

$$
\begin{aligned}
\widetilde{Q}_{2}^{\prime}= & -2 \frac{\widetilde{Q}_{2}}{s}+\frac{1}{s^{3}}\left\{-\widetilde{\mathcal{A}}_{2}+\mu R_{2,1}^{*}+\widetilde{C}_{2,2} R_{2,1}^{*}-\widetilde{D}_{2,0} \widetilde{R}_{0,1}^{*}+\widetilde{R}_{2,2}-\frac{\delta b}{(p-1)^{2}} R_{0,1}^{*}\right\} \\
& +O\left(\frac{\ln ^{2} s}{s^{4}}\right) .
\end{aligned}
$$

In fact, we now prove that there exists $\eta$ such that the order $\frac{1}{s^{3}}$ is canceled. Indeed, we choose $\eta$ such that

$$
-\widetilde{\mathcal{A}}_{2}+\mu R_{2,1}^{*}+\widetilde{C}_{2,2} R_{2,1}^{*}-\widetilde{D}_{2,0} \widetilde{R}_{0,1}^{*}+\widetilde{R}_{2,2}-\frac{\delta b}{(p-1)^{2}} R_{0,1}^{*}=0 .
$$

Using the fact that

$$
\tilde{A}_{2}=-\frac{\eta \kappa}{c_{2}},
$$

we derive

$$
\eta=-\frac{c_{2}}{\kappa}\left\{\left(-\frac{\delta b}{(p-1)^{2}}\right) R_{0,1}^{*}+\left(\mu+\widetilde{C}_{2,2}\right) R_{2,1}^{*}-\widetilde{D}_{2,0} \widetilde{R}_{0,1}^{*}+\widetilde{R}_{2,2}\right\} .
$$

The explicit formula for $\eta$ will be given by equation (93) in Appendix C. Finally, we obtain the following ODE:

$$
\widetilde{Q}_{2}^{\prime}=-\frac{2}{s} \widetilde{Q}_{2}+O\left(\frac{A^{8} \ln s^{2}}{s^{4}}\right)
$$

which implies of Proposition 3.9 (iii).

For the other estimates, we kindly refer readers to [24, Prop. 4.6] and [10, Prop. 4.10], where they can be found. Therefore, we finish our proof here.

3.2.2. The infinite-dimensional part: $\boldsymbol{q}_{-}$. The proof is similar to [24, Sect. 5.2]. So, we will sketch the main step and readers can find the details in [24]. Using the definition of the projection $P_{-}$, defined in (32), we apply it to equation (18):

$$
\begin{aligned}
P_{-}\left(\frac{\partial q}{\partial s}\right)= & P_{-}\left(\frac{\partial q}{\partial s}\right)+P_{-}\left[-i\left(\frac{\mu}{s}-\eta \frac{\ln s}{s^{2}}+\frac{\eta}{s^{2}}+\theta^{\prime}(s)\right) q\right] \\
& +P_{-}\left(V_{1} q+V_{2} \bar{q}\right)+P_{-}(B(q, y, s))+P_{-}\left(R^{*}\left(\theta^{\prime}, y, s\right)\right) .
\end{aligned}
$$

In particular, we obtain the following:

First term: $\frac{\partial q}{\partial s}$. From (33), its projection is

$$
P_{-}\left(\frac{\partial q}{\partial s}\right)=\frac{\partial q_{-}}{\partial s} .
$$

Second term: $\tilde{\mathscr{L}}_{\beta, \delta} q$. From (18) we have

$$
P_{-}\left(\mathscr{L}_{\beta, \delta} q\right)=\mathscr{L}_{\beta} q_{-}+P_{-}\left[(1+i \delta) \operatorname{Re} q_{-}\right] .
$$


Third term: $-i\left(\frac{\mu}{s}-\eta \frac{\ln s}{s^{2}}+\frac{\eta}{s^{2}}+\theta^{\prime}(s)\right) q$. Since $P_{-}$commutes with multiplication by $i$, we deduce that

$$
P_{-}\left[-i\left(\frac{\mu}{s}-\eta \frac{\ln s}{s^{2}}+\frac{\eta}{s^{2}}+\theta^{\prime}(s)\right) q\right]=-i\left(\frac{\mu}{s}-\eta \frac{\ln s}{s^{2}}+\frac{\eta}{s^{2}}+\theta^{\prime}(s)\right) q_{-} .
$$

Fourth term: $V_{1} q$ and $V_{2} \bar{q}$. We have

$$
\left\|\frac{P_{-}\left(V_{1} q\right)}{1+|y|^{M+1}}\right\|_{L^{\infty}} \leq\left\|V_{1}\right\|_{L^{\infty}}\left\|\frac{q_{-}}{1+|y|^{M+1}}\right\|_{L^{\infty}}+C \frac{A^{M}}{s^{\frac{M+2}{2}}}
$$

and

$$
\left\|\frac{V_{2} \bar{q}}{1+|y|^{M}}\right\|_{L^{\infty}} \leq\left\|V_{2}\right\|_{L^{\infty}}\left\|\frac{q-}{1+|y|^{M}}\right\|_{L^{\infty}}+C \frac{A^{M}}{s^{\frac{M+2}{2}}} .
$$

Fifth term: $B(q, y, s)$. Using (20) we have the following estimate from Lemmas A.3 and 3.15:

$$
\left\|\frac{P_{-}(B(q, y, s))}{1+|y|^{M+1}}\right\|_{L^{\infty}} \leq C(M)\left[\left(\frac{A^{M+2}}{s^{\frac{1}{2}}}\right)^{\bar{p}}+\frac{A^{5+(M+1)^{2}}}{s}\right] \frac{1}{S^{\frac{M+1}{2}}},
$$

where $\bar{p}=\min (p, 2)$.

Sixth term: $R^{*}\left(\theta^{\prime}, y, s\right)$. Using the fact that $\theta^{\prime}(s) \leq \frac{C A^{10} \ln ^{2} s}{s^{4}}$, the following holds:

$$
\left\|\frac{P_{-}\left(R^{*}\left(\theta^{\prime}, y, s\right)\right)}{1+|y|^{M+1}}\right\| \leq \frac{C}{s^{\frac{M+3}{2}}} .
$$

Using (82) and Duhamel's integral equation, we get for all $s \in\left[\tau, s_{1}\right]$,

$$
\begin{aligned}
q_{-}(s)= & e^{(s-\tau) \mathscr{L}_{\beta}} q_{-}(\tau) \\
& +\int_{\tau}^{s} e^{\left(s-s^{\prime}\right) \mathscr{L}_{\beta}} P_{-}\left[(1+i \delta) \operatorname{Re} q_{-}\right] d s^{\prime} \\
& +\int_{\tau}^{s} e^{\left(s-s^{\prime}\right) \mathscr{L}_{\beta}} P_{-}\left[-i\left(\frac{\mu^{\prime}}{s}-\frac{\eta \ln s^{\prime}}{\left(s^{\prime}\right)^{2}}+\frac{\eta}{s^{2}}+\theta^{\prime}\left(s^{\prime}\right)\right) q\right] d s^{\prime} \\
& +\int_{\tau}^{s} e^{\left(s-s^{\prime}\right) \mathscr{L}_{\beta}} P_{-}\left[V_{1} q+V_{2} \bar{q}+B\left(q, y, s^{\prime}\right)+R^{*}\left(\theta^{\prime}, y, s^{\prime}\right)\right] d s^{\prime} .
\end{aligned}
$$

Using Lemma A.2, we get

$$
\begin{aligned}
& \left\|\frac{q-(s)}{1+|y|^{M+1}}\right\|_{L^{\infty}} \leq e^{-\frac{M+1}{2}(s-\tau)}\left\|\frac{q-(\tau)}{1+|y|^{M+1}}\right\|_{L^{\infty}} \\
& \quad+\int_{\tau}^{s} e^{-\frac{M+1}{2}\left(s-s^{\prime}\right)} \sqrt{1+\delta^{2}}\left\|\frac{q_{-}}{1+|y|^{M+1}}\right\|_{L^{\infty}} d s^{\prime} \\
& \quad+\int_{\tau}^{s} e^{-\frac{M+1}{2}\left(s-s^{\prime}\right)}\left\|\frac{P_{-}\left[-i\left(\frac{\mu^{\prime}}{s}-\frac{\eta \ln s^{\prime}}{\left(s^{\prime}\right)^{2}}+\frac{\eta}{s^{2}}+\theta^{\prime}\left(s^{\prime}\right)\right) q\right]}{1+|y|^{M+1}}\right\|_{L^{\infty}} d s^{\prime} \\
& \quad+\int_{\tau}^{s} e^{-\frac{M+1}{2}\left(s-s^{\prime}\right)}\left\|\frac{P_{-}\left[V_{1} q+V_{2} \bar{q}+B\left(q, y, s^{\prime}\right)+R^{*}\left(\theta^{\prime}, y, s^{\prime}\right)\right]}{1+|y|^{M+1}}\right\|_{L^{\infty}} d s^{\prime} .
\end{aligned}
$$


By using the above estimates, we derive

$$
\begin{aligned}
& \left\|\frac{q_{-}(s)}{1+|y|^{M+1}}\right\|_{L^{\infty}} \leq e^{-\frac{M+1}{2}(s-\tau)}\left\|\frac{q_{-}(\tau)}{1+|y|^{M+1}}\right\|_{L^{\infty}} \\
& \quad+\int_{\tau}^{s} e^{-\frac{M+1}{2}\left(s-s^{\prime}\right)}\left(\sqrt{1+\delta^{2}}+\left\|\left|V_{1}\right|+\left|V_{2}\right|\right\|_{L^{\infty}}\right)\left\|\frac{q_{-}}{1+|y|^{M+1}}\right\|_{L^{\infty}} d s^{\prime} \\
& \quad+C(M) \int_{\tau}^{s} e^{-\frac{M+1}{2}\left(s-s^{\prime}\right)}\left[\frac{A^{(M+1)^{2}+5}}{\left(s^{\prime}\right)^{\frac{M+3}{2}}}+\frac{A^{(M+2) \bar{p}}}{\left(s^{\prime}\right)^{\frac{\bar{p}-1}{2}}} \frac{1}{\left(s^{\prime}\right)^{\frac{M+2}{2}}}+\frac{A^{M}}{\left(s^{\prime}\right)^{\frac{M+2}{2}}}\right] d s^{\prime} .
\end{aligned}
$$

Since we have already fixed $M$ in (30) such that

$$
M \geq 4\left(\sqrt{1+\delta^{2}}+1+2 \max _{i=1,2, y \in \mathbb{R}, s \geq 1}\left|V_{i}(y, s)\right|\right),
$$

using Gronwall's lemma, we deduce that

$$
\begin{aligned}
e^{\frac{M+1}{2} s}\left\|\frac{q_{-}(s)}{1+|y|^{M+1}}\right\|_{L^{\infty}} \leq & e^{\frac{M+1}{4}(s-\tau)} e^{\frac{M+1}{2} \tau}\left\|\frac{q_{-}(\tau)}{1+|y|^{M+1}}\right\|_{L^{\infty}} \\
& +e^{\frac{M+1}{2} s} 2^{\frac{M+3}{4}}\left[\frac{A^{(M+1)^{2}+5}}{s^{\frac{M+3}{4}}}+\frac{A^{(M+2) \bar{p}}}{s^{\frac{\bar{p}-1}{2}}} \frac{1}{\left(s^{\prime}\right)^{\frac{M+2}{2}}}+\frac{A^{M}}{s^{\frac{M+2}{2}}}\right],
\end{aligned}
$$

which concludes the proof of the last but one identity in Proposition 3.9 (iv).

3.2.3. The outer region: $\boldsymbol{q}_{\boldsymbol{e}}$. As a matter of fact, our shrinking set $\mathcal{V}_{A}(s)$ is similar to [24]. In particular, the estimate of $q_{e}$ is exactly the same. For that reason, again we omit the detailed computation. Below we give the main idea; more details can be found in [24].

In fact, using that $q(s) \in \mathcal{V}_{A}(s)$ for all $s \in\left[\tau, s_{1}\right]$, and Proposition 3.9 (i), we derive the following rough estimates:

$$
\|q(s)\|_{L^{\infty}\left(|y| \leq 2 K s^{1 / 2}\right)} \leq C \frac{A^{M+1}}{s^{1 / 2}} \quad \text { and } \quad\left|\theta^{\prime}(s)\right| \leq \frac{C A^{10} \ln ^{2} s}{s^{4}} .
$$

In particular, using the definition of $q_{e}$, given as in (17), we have

$$
\begin{aligned}
\frac{\partial q_{e}}{\partial s}= & \mathscr{L}_{\beta} q_{e}-\frac{1}{p-1} q_{e}+(1-\chi) e^{\frac{i \delta}{p-1} s}\left\{L\left(q, \theta^{\prime}, y, s\right)+R^{*}\left(\theta^{\prime}, y, s\right)\right\} \\
& -e^{\frac{i \delta}{p-1} s} q(s)\left(\partial_{s} \chi+(1+i \beta) \Delta \chi+\frac{1}{2} y \cdot \nabla \chi\right) \\
& +2 e^{\frac{i \delta}{p-1} s}(1+i \beta) \operatorname{div}(q(s) \nabla \chi) .
\end{aligned}
$$

In addition to this, we can write (85) under Duhamel's integral equation and take an $L^{\infty}$ estimate:

$$
\begin{aligned}
\left\|q_{e}(s)\right\|_{L^{\infty}} \leq & e^{-\frac{s-\tau}{p-1}}\left\|q_{e}(\tau)\right\|_{L^{\infty}}, \\
& +\int_{\tau}^{s} e^{-\frac{s-s^{\prime}}{p-1}}\left(\left\|(1-\chi) L\left(q, \theta^{\prime}, y, s^{\prime}\right)\right\|_{L^{\infty}}+\left\|(1-\chi) R^{*}\left(\theta^{\prime}, y, s^{\prime}\right)\right\|_{L^{\infty}}\right) d s^{\prime} \\
& +\int_{\tau}^{s} e^{-\frac{s-s^{\prime}}{p-1}}\left\|q\left(s^{\prime}\right)\left(\partial_{s} \chi+(1+i \beta) \Delta \chi+\frac{1}{2} y \cdot \nabla \chi\right)\right\|_{L^{\infty}} d s^{\prime} \\
& +\int_{\tau}^{s} e^{-\frac{s-s^{\prime}}{p-1}} \frac{1}{\sqrt{1-e^{-\left(s-s^{\prime}\right)}}}\left\|q\left(s^{\prime}\right) \nabla \chi\right\|_{L^{\infty}} d s^{\prime}
\end{aligned}
$$


Thanks to detailed computation given in of [24, Sect. 5.3], we obtain

$$
\begin{aligned}
\left\|q_{e}(s)\right\|_{L^{\infty}} \leq & e^{-\frac{s-\tau}{p-1}}\left\|q_{e}(\tau)\right\|_{L^{\infty}} \\
& +\int_{\tau}^{s} e^{-\frac{s-s^{\prime}}{p-1}}\left(\frac{1}{2(p-1)}\left\|q_{e}\left(s^{\prime}\right)\right\|_{L^{\infty}}+C \frac{A^{M+1}}{\sqrt{s^{\prime}}}\right. \\
& \left.\quad+C \frac{A^{M+1}}{s^{\prime}} \frac{1}{\sqrt{1-e^{-\left(s-s^{\prime}\right)}}}\right) d s^{\prime}
\end{aligned}
$$

By using Gronwall's inequality, we derive

$$
\left\|q_{e}(s)\right\|_{L^{\infty}} \leq e^{-\frac{(s-\tau)}{2(p-1)}}\left\|q_{e}(\tau)\right\|_{L^{\infty}}+\frac{C A^{M+1}}{\tau^{\frac{1}{4}}}(s-\tau+\sqrt{s-\tau}),
$$

which yields the proof of Proposition 3.9 (iv).

\subsection{The case $\beta=0$}

Here we give an argument for the special case where $\beta=0$. The main reasoning comes from Definition 3.1 for $\mathcal{V}_{A}(s)$, in particular (41). In particular, there is only one bound that becomes singular: $\tilde{A}_{2}=-\frac{R_{0,1}^{*}}{c_{2}}$. Naturally, we change this bound to a new one:

$$
\left|\widetilde{Q}_{2}\right|=\left|\tilde{q}_{2}-\left(\frac{\tilde{A}_{2} \ln s}{s^{2}}+\frac{\widetilde{B}_{2}}{s^{2}}\right)\right| \leq \frac{A^{10} \ln ^{2} s}{s^{3}},
$$

where $\tilde{A}_{2}$ is defined by (41) and $\widetilde{B}_{2}$ can be chosen arbitrarily. In addition to this, we also denote the new shrinking set by $\mathcal{V}_{A}(s)$. In particular, Proposition 3.9 remains valid, except the ODEs for $\theta^{\prime}(s)$ and $\widetilde{Q}_{2}$.

- For $\theta^{\prime}(s)$ : Repeating the process for the case $\beta \neq 0$, we derive

$$
\left|\kappa \theta^{\prime}(s)-\left(\frac{R_{0,1}^{*}}{s^{2}}+\frac{T_{0,1}^{*} \ln s}{s^{2}}\right)\right| \leq \frac{C \ln s}{s^{3}} .
$$

When $\beta=0, R_{0,1}^{*}=0$. However, the leading order $\frac{T_{0,1}^{*} \ln s}{s^{2}}$ will generate

$$
\theta(s) \sim \frac{\theta_{0} \ln s}{s}
$$

This violates our purpose that

$$
\theta(s) \ll \frac{\ln s}{s} .
$$

Hence, it imposes

$$
\eta=0
$$

Note that constants $T_{i, j}^{*}, \widetilde{T}_{i, j}^{*}=0$. Thus, we get

$$
\left|\kappa \theta^{\prime}(s)-\frac{R_{0,1}^{*}}{s^{2}}\right| \leq \frac{C \ln s}{s^{3}} .
$$


It is sufficient to prove Proposition 3.9 (iii). Indeed, we take the projection of equation (18) on $\tilde{h}_{2}$, as in equation (77). In particular, plugging (86) into

$$
\widetilde{P}_{2, M}\left(R^{*}\right)=\frac{\widetilde{R}_{2,2}^{*}}{s^{3}}+\frac{\theta^{\prime}(s) \kappa}{s} \frac{-\delta b}{(p-1)^{2}}+O\left(\frac{\ln s}{s^{4}}\right),
$$

we obtain

$$
\begin{aligned}
\widetilde{P}_{2, M}\left(R^{*}\right) & =\frac{\widetilde{R}_{2,2}^{*}}{s^{3}}+\left(\frac{R_{0,1}^{*}}{s^{2}}\right) \frac{-\delta b}{s(p-1)^{2}}+O\left(\frac{\ln s}{s^{4}}\right) \\
& =\left(\widetilde{R}_{2,2}^{*}-\frac{\delta b R_{0,1}^{*}}{(p-1)^{2}}\right) \frac{1}{s^{3}}+O\left(\frac{\ln s}{s^{4}}\right) .
\end{aligned}
$$

Note that, when $\beta=0$ we have

$$
\delta \mu+\widetilde{D}_{2,2}=-2
$$

and

$$
\widetilde{T}_{2,2}^{*}-\frac{T_{0,1}^{*} \delta b}{(p-1)^{2}}=0
$$

Hence we have

$$
\begin{aligned}
\tilde{q}_{2}^{\prime}= & -\frac{2 \tilde{q}_{2}}{s}+\frac{1}{s^{3}}\left\{\mu R_{2,1}^{*}+\widetilde{C}_{2,2} R_{2,1}^{*}-\widetilde{D}_{2,0} \widetilde{R}_{0,1}^{*}+\widetilde{R}_{2,2}-\frac{\delta b}{(p-1)^{2}} R_{0,1}^{*}\right\} \\
& +O\left(\frac{\ln ^{2} s}{s^{4}}\right) .
\end{aligned}
$$

Using the decomposition $\widetilde{Q}_{2}=\tilde{q}_{2}-\left(\frac{\tilde{\mathscr{A}}_{2}(\beta=0) \ln s}{s^{2}}+\frac{\widetilde{\mathscr{B}}_{2}(\beta=0)}{s^{2}}\right)$, then $\widetilde{Q}_{2}$ reads

$$
\begin{aligned}
\widetilde{Q}_{2}= & -\frac{2}{s} \widetilde{Q}_{2}+\left(-\widetilde{\mathcal{A}}_{2}+\mu R_{2,1}^{*}+\widetilde{C}_{2,2} R_{2,1}^{*}-\widetilde{D}_{2,0} \widetilde{R}_{0,1}^{*}+\widetilde{R}_{2,2}-\frac{\delta b}{(p-1)^{2}} R_{0,1}^{*}\right) \frac{1}{s^{3}} \\
& +O\left(\frac{\ln ^{2} s}{s^{4}}\right) .
\end{aligned}
$$

Note that it is not similar to the case $\beta \neq 0$; the role of $\eta$ vanishes. The order $\frac{1}{s^{3}}$ is canceled by

$$
\tilde{A}_{2}=\mu R_{2,1}^{*}+\widetilde{C}_{2,2} R_{2,1}^{*}-\widetilde{D}_{2,0} \widetilde{R}_{0,1}^{*}+\widetilde{R}_{2,2}-\frac{\delta b}{(p-1)^{2}} R_{0,1}^{*} .
$$

In particular, when $\beta=\delta=0$ we can explicitly compute

$$
\tilde{\mathcal{A}}_{2}=-\widetilde{C}_{2,2} R_{2,1}^{*}+\widetilde{R}_{2,2}^{*}=-\left(-\frac{1}{4}\right) \frac{5 \kappa}{8 p}-\frac{5}{32} \kappa \frac{(5 p-4)}{p^{2}}=-\frac{5 \kappa(p-1)}{8 p^{2}} .
$$

This constant exactly matches the formal approach given by Velázquez, Galaktionov and Herrero [37]. 


\section{A. Spectral properties of $\mathscr{L}_{\beta}$}

In this appendix, we recall from [24, App. A] some properties associated to the operator $\mathscr{L}_{\beta}$, defined in (15). We recall that

$$
\mathscr{L}_{\beta} v=(1+i \beta) \Delta v-\frac{1}{2} y \cdot \nabla v=\frac{1}{\rho_{\beta}} \operatorname{div}\left(\rho_{\beta} \nabla w\right),
$$

where

$$
\rho_{\beta}(y)=\frac{e^{-\frac{|y|^{2}}{4(1+i \beta)}}}{(4 \pi(1+i \beta))^{N / 2}} .
$$

Moreover, the operator $\mathscr{L}_{\beta}$ is self-adjoint with respect to the weight $\rho_{\beta}$ in the sense that

$$
\int_{\mathbb{R}^{N}} u(y) \mathscr{L}_{\beta} w(y) \rho_{\beta}(y) d y=\int_{\mathbb{R}^{N}} w(y) \mathscr{L}_{\beta} u(y) \rho_{\beta}(y) d y .
$$

In one space dimension $(N=1)$, the eigenfunctions $f_{n}$ of $\mathscr{L}_{\beta}$ are dilations of standard Hermite polynomials $H_{n}(y)$ :

$$
f_{n}(y)=H_{n}\left(\frac{y}{2 \sqrt{1+i \beta}}\right), \quad \text { where } \mathscr{L}_{\beta} H_{n}=-\frac{n}{2} H_{n} .
$$

If $N \geq 2$, its eigenfunction $f_{\alpha}\left(y_{1}, \ldots, y_{N}\right)$, where $\alpha=\left(\alpha_{1}, \ldots, \alpha_{N}\right) \in \mathbb{N}^{N}$ is a multiindex, are given by

$$
f_{\alpha}(y)=\prod_{i=1}^{N} f_{\alpha_{i}}\left(y_{i}\right)=\prod_{i=1}^{N} H_{\alpha_{i}}\left(\frac{y_{i}}{2 \sqrt{1+i \beta}}\right) .
$$

The family $f_{\alpha}$ is orthogonal in the sense that for all $\alpha$ and $\xi \in \mathbb{N}^{N}$,

$$
\int f_{\alpha} f_{\xi} \rho_{\beta} d y=\delta_{\alpha, \xi} \int f_{\alpha}^{2} \rho_{\beta} d y \text {. }
$$

The semigroup generated by $\mathscr{L}_{\beta}$ is well defined and has the kernel

$$
e^{s \mathscr{L}_{\beta}}(y, x)=\frac{1}{\left[4 \pi(1+i \beta)\left(1-e^{-s}\right)\right]^{N / 2}} \exp \left[-\frac{\left|x-y e^{-\frac{s}{2}}\right|^{2}}{4(1+i \beta)\left(1-e^{-s}\right)}\right] .
$$

In the following, we give some properties associated to the kernel.

\section{Lemma A.1.}

(a) The semigroup associated to $\mathscr{L}_{\beta}$ satisfies the maximum principle

$$
\left\|e^{s \mathscr{L}_{\beta}} \varphi\right\|_{L^{\infty}} \leq\|\varphi\|_{L^{\infty}} .
$$

(b) Moreover, we have

$$
\left\|e^{s \mathscr{L}_{\beta}} \operatorname{div}(\varphi)\right\|_{L^{\infty}} \leq \frac{C}{\sqrt{1-e^{-s}}}\|\varphi\|_{L^{\infty}},
$$

where $C$ only depends on $\beta$. 
Proof.

(a) The result directly follows from the definition of the semigroup given in (89).

(b) Using integration by parts and (a), the conclusion follows.

Lemma A.2. There exists a constant $C$ such that if $\phi$ satisfies

$$
\text { for all } x \in \mathbb{R}, \quad|\phi(x)| \leq\left(1+|x|^{M+1}\right),
$$

then for all $y \in \mathbb{R}$ we have

$$
\left|e^{s \mathscr{L}_{\beta}} P_{-}(\phi(y))\right| \leq C e^{-\frac{M+1}{2} s}\left(1+|y|^{M+1}\right) .
$$

Proof. This also follows directly from the definition of the semigroup, through integration by parts; for a similar case see [4, pp. 556-558].

Moreover, we have the following useful lemma concerning $P_{-}$.

Lemma A.3. For all $k \geq 0$ we have

$$
\left\|\frac{P_{-}(\phi)}{1+|y|^{M+k}}\right\|_{L^{\infty}} \leq C\left\|\frac{\phi}{1+|y|^{M+k}}\right\| .
$$

Proof. Using (31) we have

$$
\left|\phi_{n}\right| \leq C\left\|\frac{\phi}{1+|y|^{M+k}}\right\|_{L^{\infty}} .
$$

Since for all $m \leq M$, we have $\left|h_{m}(y)\right| \leq C\left(1+|y|^{m+k}\right)$ and

$$
|\phi| \leq C\left\|\frac{\phi}{1+|y|^{M+k}}\right\|_{L^{\infty}}\left(1+|y|^{m+k}\right),
$$

the result follows from definition (29) of $\phi$.

\section{B. Details of expansions of the potential terms: $V_{1}$ and $V_{2}$}

In this section we aim to give expansions of $V_{1}$ and $V_{2}$ in order to give the conclusion of Lemma 3.14 (i) and some related constants. Indeed, we recall the definitions of $V_{1}$ and $V_{2}$ :

$$
\begin{aligned}
& V_{1}(y, s)=(1+i \delta) \frac{p+1}{2}\left(|\varphi|^{p-1}-\frac{1}{p-1}\right), \\
& V_{2}(y, s)=(1+i \delta) \frac{p-1}{2}\left(|\varphi|^{p-3} \varphi^{2}-\frac{1}{p-1}\right),
\end{aligned}
$$


where

$$
\varphi(y, s)=\varphi_{0}(y, s)+\frac{(1+i \delta) a}{s}=\kappa\left(1+\frac{b}{p-1} \frac{|y|^{2}}{s}\right)^{-\frac{1+i \delta}{p-1}}+\frac{(1+i \delta) a}{s}
$$

and

$$
a=\frac{2 \kappa b(1-\delta \beta)}{(p-1)^{2}} .
$$

Then, using Taylor expansion, we claim the following asymptotic behavior:

$$
V_{1}(y, s)=\frac{1}{s} W_{1,1}(y)+\frac{1}{s^{2}} W_{1,2}+O\left(\frac{1+|y|^{6}}{s^{3}}\right)
$$

and

$$
V_{2}(y, s)=\frac{1}{s} W_{2,1}(y)+\frac{1}{s^{2}} W_{2,2}(y)+O\left(\frac{1+|y|^{6}}{s^{3}}\right),
$$

where

$$
\begin{aligned}
W_{1,1}(y)= & (1+i \delta) \frac{(p+1)}{2} \frac{b}{(p-1)^{2}}\left(-y^{2}+2(1-\delta \beta)\right), \\
W_{1,2}(y)= & (1+i \delta) \frac{(p+1)}{2} \frac{b^{2}}{(p-1)^{3}}\left\{y^{4}-\frac{\left(2(1-\delta \beta)\left(p-2+\delta^{2}\right)\right)}{p-1} y^{2}\right. \\
& \left.+\frac{(p-1)\left(1+\delta^{2}\right)(1-\delta \beta)^{2}+(p-3)\left(1-\delta^{2}\right)(1-\delta \beta)^{2}}{p-1}\right\} \\
= & (1+i \delta) \frac{(p+1)}{2} \frac{b^{2}}{(p-1)^{4}}\left\{(p-1) y^{4}-\left[2(1-\delta \beta)\left(p-2+\delta^{2}\right)\right] y^{2}\right. \\
& \left.+2\left(p-2+\delta^{2}\right)(1-\delta \beta)^{2}\right\}
\end{aligned}
$$

and

$$
\begin{aligned}
W_{2,1}(y)= & (1+i \delta) \frac{b}{2(p-1)^{2}}\left\{(p-1+2 i \delta)\left(-y^{2}+2(1-\delta \beta)\right)\right\}, \\
W_{2,2}(y)= & (1+i \delta) \frac{b^{2}}{2(p-1)^{4}}\left\{(p-1+2 i \delta)(p-1+i \delta) y^{4}\right. \\
& -\left(2(p-1)(p-2)+(2 p-10) \delta^{2}+(8 p-16) \delta i\right)(1-\delta \beta) y^{2} \\
& +(1-\delta \beta)^{2}\left[\frac{(p+1)(p-1)(1+i \delta)^{2}}{2}+(p+1)(p-3)\left(1+\delta^{2}\right)\right. \\
& \left.\left.+\frac{(p-3)(p-5)(1-i \delta)^{2}}{2}\right]\right\} .
\end{aligned}
$$

For the proofs of (90) and (91), we kindly refer readers to [10, Appendix B].

In addition to this, we aim to determine the constants given in Lemma 3.14 (ii):

$$
\widetilde{D}_{4,2}=\widetilde{P}_{4, M}\left(W_{1,1} \tilde{h}_{2}+W_{2,1} \overline{\tilde{h}}_{2}\right)=\frac{b\left(\delta^{2}-p\right)}{(p-1)^{2}},
$$




$$
\begin{aligned}
& D_{2,2}=P_{2, M}\left(W_{1,1} \tilde{h}_{2}+W_{2,1} \overline{\tilde{h}}_{2}\right) \\
& =-\frac{b}{2(p-1)^{2}}\left\{-24 p \delta+56 \delta^{3}+64 \delta^{2} \beta+32 \delta+24 p \delta^{2} \beta+40 \delta^{4} \beta\right\}, \\
& \tilde{L}_{2,4}=\widetilde{P}_{2, M}\left(i \tilde{h}_{4}\right)=6 \delta^{2} \beta-12 \delta-6 \beta, \\
& D_{4,2}=P_{4, M}\left(W_{1,1} \tilde{h}_{2}+W_{2,1} \overline{\tilde{h}}_{2}\right)=\frac{b}{(p-1)^{2}}\left\{-2 \delta\left(1+\delta^{2}\right)\right\}, \\
& \widetilde{D}_{2,0}=\widetilde{P}_{2, M}\left(W_{1,1} \tilde{h}_{0}+W_{2,1} \overline{\tilde{h}}_{0}\right)=-\frac{b}{2(p-1)^{2}}\left(2 p-2 \delta^{2}\right), \\
& \tilde{L}_{0,2}=\widetilde{P}_{0, M}\left(i \tilde{h}_{2}\right)=-2 \delta+\delta^{2} \beta-\beta, \\
& \widetilde{D}_{0,2}=\widetilde{P}_{0, M}\left(W_{1,1} \tilde{h}_{2}+W_{2,1} \overline{\tilde{h}}_{2}\right) \\
& =-\frac{b}{2(p-1)^{2}}\left\{-32 \delta \beta-12 p \beta^{2}+12 \delta^{2} \beta^{2}-16 \delta^{2}+16 p-4 \delta^{4} \beta^{2}+4 p \delta^{2} \beta^{2}\right. \\
& -32 p \delta \beta\} \\
& \widetilde{C}_{2,2}=\widetilde{P}_{2, M}\left(W_{1,1} h_{2}+W_{2,1} \bar{h}_{2}\right) \\
& =-\frac{b}{2(p-1)^{2}}\left\{-14 \delta^{2} \beta+2 p \beta-12 \beta\right\}, \\
& \widetilde{C}_{2,4}=\widetilde{P}_{2, M}\left(W_{1,1} h_{4}+W_{2,1} \bar{h}_{4}\right) \\
& =-\frac{b}{2(p-1)^{2}}\left\{96 p \beta+224 \delta^{3} \beta^{2}-288 \delta^{2} \beta-128 p \delta \beta^{2}-192 \beta+96 \delta \beta^{2}\right\}, \\
& \widetilde{D}_{2,4}=\widetilde{P}_{2, M}\left(W_{1,1} \tilde{h}_{4}+W_{2,1} \overline{\tilde{h}}_{4}\right) \\
& =-\frac{b}{2(p-1)^{2}}\left\{-96 p \delta^{2} \beta^{2}-168 p \delta \beta+96 p-528 \delta \beta-96 \delta^{2}+216 \delta^{2} \beta^{2}\right. \\
& \left.-168 p \beta^{2}+144 \delta^{4} \beta^{2}-360 \delta^{3} \beta\right\}, \\
& \widetilde{F}_{2,2}=\widetilde{P}_{2, M}\left(W_{1,2} \tilde{h}_{2}+W_{2,2} \overline{\tilde{h}}_{2}\right) \\
& =\frac{b^{2}}{2(p-1)^{4}}\left\{-240 p+276 p^{2}-312 p \delta^{2}-204 \delta^{4}+\left(-288 p-552 p^{2}+696\right) \delta \beta\right. \\
& +(432-144 p) \delta^{3} \beta+144 \delta^{5} \beta+\left(180 p-180 p^{2}\right) \beta^{2} \\
& \left.+\left(96 p^{2}+288 p-96\right) \delta^{2} \beta^{2}+(108+36 p) \delta^{4} \beta^{2}\right\}, \\
& D_{0,2}=P_{0, M}\left(W_{1,1} \tilde{h}_{2}+W_{1,2} \overline{\tilde{h}}_{2}\right) \\
& =-\frac{b}{2(p-1)^{2}}\left\{32 \delta+24 \delta^{5} \beta^{2}+64 \delta^{2} \beta+48 \delta^{3} \beta^{2}+64 \delta^{4} \beta+32 \delta^{3}+24 \delta \beta^{2}\right. \\
& \left.+96 p \delta^{3} \beta^{2}+96 p \delta \beta^{2}\right\}, \\
& L_{0,2}=P_{0, M}\left(i \tilde{h}_{2}\right)=4 \delta \beta+4 \delta^{3} \beta \text {. }
\end{aligned}
$$




\section{Details of expansions of $R^{*}\left(y, s, \theta^{\prime}(s)\right)$}

Using the definition of $\varphi,(18)$ and the fact that $\varphi_{0}$ satisfies (13), we see that $R^{*}$ is defined as

$$
\begin{aligned}
R^{*}= & \frac{(1+i \beta)}{s} \Delta_{z} \varphi_{0}(z)+\frac{1}{2 s} z \cdot \nabla \varphi_{0}+\frac{a}{s^{2}}(1+i \delta)-\frac{(1+i \delta)^{2} a}{(p-1) s} \\
& +(1+i \delta)\left(F\left(\varphi_{0}(z)+\frac{a}{s}(1+i \delta)\right)-F\left(\varphi_{0}(z)\right)\right) \\
& -i\left(\frac{\mu}{s}-\eta \frac{\ln s}{s^{2}}+\frac{\eta}{s^{2}}+\theta^{\prime}(s)\right)\left(\varphi_{0}(z)+\frac{a}{s}(1+i \delta)\right) \\
= & R_{1}^{*}(y, s)+\frac{\ln s}{s^{2}} T_{1}+\frac{1}{s^{2}} T_{2}++\theta^{\prime}(s) \Theta(y, s),
\end{aligned}
$$

where $F(w)=|w|^{p-1} w, \Theta(y, s)=-i\left(\varphi_{0}(y, s)+\frac{a(1+i \delta)}{s}\right), T_{1}^{*}=-\eta \Theta$ and $T^{* *}=\eta \Theta$.

\section{Expansion of $R_{1}^{*}(y, s)$ in terms of $h_{j}$ and $\tilde{h}_{j}$}

As a matter of fact, we can expand $R_{1}^{*}$ in a series of $\frac{1}{s^{j}}$ as

$$
R_{1}^{*}(y, s)=\frac{1}{s} \mathcal{R}_{0}(y)+\frac{1}{s^{2}} \mathcal{R}_{1}(y)+\frac{1}{s^{3}} \mathcal{R}_{2}(y)+\widetilde{\mathcal{R}}(y, s),
$$

where $\widetilde{\mathcal{R}}$ satisfies

$$
|\widetilde{R}(y, s)| \leq \frac{C\left(1+|y|^{6}\right)}{s^{4}},
$$

which implies that

$$
\left|P_{j, M}(\widetilde{\mathcal{R}})\right|+\left|\widetilde{P}_{j, M}(\widetilde{\mathcal{R}})\right| \leq \frac{C}{s^{4}} .
$$

In addition to this, we can write $\mathcal{R}_{j}(y)$ in the basis generated by $h_{k}$, and $\tilde{h}_{k}$ as

$$
\mathcal{R}_{j}(y)=\sum_{k=0}^{j}\left(R_{j, k}^{*} h_{k}+\widetilde{R}_{j, k}^{*} \tilde{h}_{k}\right) .
$$

Repeating the method given in [10, Sect. D], we can find explicit formulas for the constants $R_{i, j}^{*}$ and $\widetilde{R}_{i, j}^{*}$. Here we give only the results:

$$
\begin{aligned}
& R_{0,0}^{*}=-\kappa\left(\mu+\frac{2 b \beta\left(1+\delta^{2}\right)}{(p-1)^{2}}\right), \\
& \widetilde{R}_{0,0}^{*}=a-\frac{2 \kappa b(1-\delta \beta)}{(p-1)^{2}}, \\
& \widetilde{R}_{2,1}^{*}=\frac{4 \kappa\left(p+(p+1) \delta \beta-\delta^{2}\right) b^{2}}{(p-1)^{4}}-\frac{\kappa b}{(p-1)^{2}}, \\
& R_{2,1}^{*}=\frac{2 \kappa b^{2}\left(\delta+3 p \beta+3 p \delta^{2} \beta-\beta+\delta^{3}+\delta^{4} \beta\right)}{(p-1)^{4}},
\end{aligned}
$$




$$
\begin{aligned}
\widetilde{R}_{0,1}^{*}= & \frac{2 \kappa b^{2}}{(p-1)^{4}}\left(3 \delta^{3} \beta+\left(2 p \beta^{2}+6 \beta^{2}-5\right) \delta^{2}+(-7 \beta-10 p \beta) \delta+5 p-3 p \beta^{2}+\beta^{2}\right), \\
R_{0,1}^{*}= & -\frac{4 \beta \kappa b^{2}}{(p-1)^{4}}\left(2 \delta^{4}+\beta \delta^{3}+3 p \delta^{2}+\beta \delta+3 p-2\right), \\
\widetilde{R}_{2,2}^{*}= & \frac{6 \kappa b^{2}}{(p-1)^{4}}\left\{\delta^{3} \beta-2 \delta^{2}-(2 p+1) \delta \beta+2 p\right\} \\
& -\frac{2 \kappa b^{3}}{(p-1)^{6}}\left\{3 \beta^{2} \delta^{6}-12 \beta \delta^{5}+\left(9-12 \beta^{2}-6 p \beta^{2}\right) \delta^{4}+(42 p \beta+42 \beta) \delta^{3}\right. \\
& +\left(70 p \beta^{2}+19 p^{2} \beta^{2}-78 p-6 \beta^{2}\right) \delta^{2} \\
& \left.\quad+\left(-98 p^{2} \beta+36 \beta-74 p \beta\right) \delta-20 p+49 p^{2}+18 p \beta^{2}-30 p^{2} \beta^{2}\right\} .
\end{aligned}
$$

We do not need to formulate constants other than these.

\section{Expansion of $\theta^{\prime}(s) \Theta(y)$}

We introduce

$$
\Theta(y, s)=-i\left(\varphi_{0}(y, s)+\frac{a(1+i \delta)}{\sqrt{s}}\right),
$$

where $\varphi_{0}$ and $a$ are defined as in (12) and (11), respectively. Using Taylor expansion we write

$$
\begin{aligned}
\Theta(y, s)= & -i \kappa+\kappa(\delta-i) \frac{y^{2}}{s} \frac{b}{(p-1)^{2}}+a(\delta-i) \frac{1}{s} \\
& +\kappa(1-i \beta) \delta(p+1) \frac{y^{4}}{s^{2}} \frac{b^{2}}{2(p-1)^{4}}+\widetilde{\Theta}(y, s),
\end{aligned}
$$

where $\widetilde{\Theta}(y, s)$ satisfies

$$
|\widetilde{\Theta}(y, s)| \leq \frac{C\left(1+|y|^{6}\right)}{s^{3}},
$$

which yields

$$
\left|P_{j, M}(\widetilde{\Theta})\right|+\left|\widetilde{P}_{j, M}(\widetilde{\Theta})\right| \leq \frac{C}{s^{3}}
$$

and

$$
\begin{aligned}
-i \kappa & +\kappa(\delta-i) \frac{y^{2}}{s} \frac{b}{(p-1)^{2}}+a(\delta-i) \frac{1}{s}+\kappa(1-i \beta) \delta(p+1) \frac{y^{4}}{s^{2}} \frac{b^{2}}{2(p-1)^{4}} \\
& =\left(-\kappa+\frac{\Theta_{0,0}^{*}}{s}\right) h_{0}+\frac{\widetilde{\Theta}_{0,0}^{*}}{s} \tilde{h}_{0}+\frac{\Theta_{2,0}^{*}}{s} h_{2}+\frac{\widetilde{\Theta}_{2,0}^{*}}{s} \tilde{h}_{2}++O\left(\frac{1+|y|^{4}}{s^{2}}\right) .
\end{aligned}
$$

In addition to this, we can calculate these constants and we obtain

$$
\begin{aligned}
& \Theta_{0,0}^{*}=4\left(1+\delta^{2}\right) \delta \beta \frac{\kappa b}{(p-1)^{2}}, \\
& \widetilde{\Theta}_{0,0}^{*}=-\beta\left(1+\delta^{2}\right) \frac{\kappa b}{(p-1)^{2}},
\end{aligned}
$$




$$
\begin{aligned}
& \widetilde{\Theta}_{2,0}^{*}=-\delta \frac{\kappa b}{(p-1)^{2}}, \\
& \Theta_{2,0}^{*}=\left(1+\delta^{2}\right) \frac{\kappa b}{(p-1)^{2}}, \\
& \widetilde{\Theta}_{2,1}^{*}=-3 \delta(p+1)\left(-\beta^{2}+\beta \delta-2\right) \frac{\kappa b^{2}}{(p-1)^{4}} .
\end{aligned}
$$

In particular, we also have the following expansions of $T^{*}=-\eta \Theta$ and $T^{* *}=\eta \Theta$ :

$$
\begin{aligned}
T^{*} & =\left(\eta \kappa+\frac{T_{0,0}^{*}}{s}\right) h_{0}+\frac{\tilde{T}_{0,0}^{*}}{s} \tilde{h}_{0}+\frac{T_{2,0}^{*}}{s} h_{2}+\frac{\tilde{T}_{2,0}^{*}}{s} \tilde{h}_{2}++O\left(\frac{1+|y|^{4}}{s^{2}}\right), \\
T^{* *} & =\left(-\eta \kappa+\frac{T_{0,0}^{*}}{s}\right) h_{0}+\frac{\tilde{T}_{0,0}^{*}}{s} \tilde{h}_{0}+\frac{T_{2,0}^{*}}{s} h_{2}+\frac{\tilde{T}_{2,0}^{*}}{s} \tilde{h}_{2}+O\left(\frac{1+|y|^{4}}{s^{2}}\right),
\end{aligned}
$$

where

$$
\left(T_{i, j}^{*}, \widetilde{T}_{i, j}^{*}\right)=-\eta\left(\Theta_{i, j}^{*}, \widetilde{\Theta}_{i, j}^{*}\right) \quad \text { and } \quad T_{i, j}^{* *}, \widetilde{T}_{i, j}^{* *}=\eta\left(\Theta_{i, j}^{*}, \widetilde{\Theta}_{i, j}^{*}\right) .
$$

Finally, we aim to give the explicit form of $\eta$ here: indeed, we have the following formula from (81):

$$
\eta=-\frac{c_{2}}{\kappa}\left\{\left(-\frac{\delta b}{(p-1)^{2}}\right) R_{0,1}^{*}+\left(\mu+\widetilde{C}_{2,2}\right) R_{2,1}^{*}-\widetilde{D}_{2,0} \widetilde{R}_{0,1}^{*}+\widetilde{R}_{2,2}\right\} .
$$

Using the formulas for the constants in $\eta$, we obtain

$$
\begin{aligned}
\eta=- & \frac{\beta\left(1+\delta^{2}\right)}{8\left(p-(p+1) \delta \beta-\delta^{2}\right)^{3}} \\
\times & \left\{\delta^{6} \beta^{2}+3 \delta^{5} \beta+\left(3 \beta^{2} p+10\right) \delta^{4}+(5 \beta+18 p \beta) \delta^{3}\right. \\
& +\left(2 \beta^{2} p^{2}+7 \beta^{2}+10 p+\beta^{2} p\right) \delta^{2}+\left(-18 \beta+18 p \beta+20 p^{2} \beta\right) \delta \\
& \left.+10 p-2 \beta^{2}+12 \beta^{2} p^{2}-2 \beta^{2} p-10 p^{2}\right\} .
\end{aligned}
$$

\section{References}

[1] B. Abdelhedi and H. Zaag, Construction of a blow-up solution for a perturbed nonlinear heat equation with a gradient and a non-local term. J. Differential Equations 272 (2021), 1-45

Zbl 1454.35221 MR 4156118

[2] I. S. Aranson and L. Kramer, The world of the complex Ginzburg-Landau equation. Rev. Modern Phys. 74 (2002), no. 1, 99-143 Zbl 1205.35299 MR 1895097

[3] M. Berger and R. V. Kohn, A rescaling algorithm for the numerical calculation of blowing-up solutions. Comm. Pure Appl. Math. 41 (1988), no. 6, 841-863 Zbl 0652.65070 MR 948774

[4] J. Bricmont and A. Kupiainen, Universality in blow-up for nonlinear heat equations. Nonlinearity 7 (1994), no. 2, 539-575 Zbl 0857.35018 MR 1267701 
[5] T. Cazenave, Semilinear Schrödinger equations. Courant Lect. Notes Math. 10, New York University, Courant Institute of Mathematical Sciences, New York; American Mathematical Society, Providence, RI, 2003 MR 2002047

[6] R. Côte and H. Zaag, Construction of a multisoliton blowup solution to the semilinear wave equation in one space dimension. Comm. Pure Appl. Math. 66 (2013), no. 10, 1541-1581 Zbl 1295.35124 MR 3084698

[7] G. K. Duong, A blowup solution of a complex semi-linear heat equation with an irrational power. J. Differential Equations 267 (2019), no. 9, 4975-5048 Zbl 1428.35143 MR 3991552

[8] G. K. Duong, Profile for the imaginary part of a blowup solution for a complex-valued semilinear heat equation. J. Funct. Anal. 277 (2019), no. 5, 1531-1579 Zbl 1417.35064 MR 3969198

[9] G. K. Duong, V. T. Nguyen, and H. Zaag, Construction of a stable blowup solution with a prescribed behavior for a non-scaling-invariant semilinear heat equation. Tunis. J. Math. 1 (2019), no. 1, 13-45 Zbl 1407.35092 MR 3907732

[10] G. K. Duong, N. Nouaili, and H. Zaag, Construction of blow-up solutions for the complex Ginzburg-Landau equation with critical parameters. Mem. Amer. Math. Soc., 2020, to appear.

[11] G. K. Duong and H. Zaag, Profile of a touch-down solution to a nonlocal MEMS model. Math. Models Methods Appl. Sci. 29 (2019), no. 7, 1279-1348 Zbl 1425.35116 MR 3974168

[12] M. A. Ebde and H. Zaag, Construction and stability of a blow up solution for a nonlinear heat equation with a gradient term. SeMA J. (2011), no. 55, 5-21 Zbl 1241.35125 MR 2849015

[13] T.-E. Ghoul, V. T. Nguyen, and H. Zaag, Blowup solutions for a nonlinear heat equation involving a critical power nonlinear gradient term. J. Differential Equations 263 (2017), no. 8, 4517-4564 Zbl 1378.35173 MR 3680932

[14] T.-E. Ghoul, V. T. Nguyen, and H. Zaag, Blowup solutions for a reaction-diffusion system with exponential nonlinearities. J. Differential Equations 264 (2018), no. 12, 7523-7579 Zbl 1393.35096 MR 3779644

[15] T.-E. Ghoul, V. T. Nguyen, and H. Zaag, Construction and stability of blowup solutions for a non-variational semilinear parabolic system. Ann. Inst. H. Poincaré Anal. Non Linéaire 35 (2018), no. 6, 1577-1630 Zbl 1394.35222 MR 3846237

[16] T.-E. Ghoul, V. T. Nguyen, and H. Zaag, Construction and stability of type I blowup solutions for non-variational semilinear parabolic systems. Adv. Pure Appl. Math. 10 (2019), no. 4, 299312 Zbl 1425.35108 MR 4015203

[17] T.-E. Ghoul, V. T. Nguyen, and H. Zaag, Construction of type I blowup solutions for a higher order semilinear parabolic equation. Adv. Nonlinear Anal. 9 (2020), no. 1, 388-412 Zbl 1416.35141 MR 3962631

[18] J. Ginibre and G. Velo, The Cauchy problem in local spaces for the complex Ginzburg-Landau equation. In Differential equations, asymptotic analysis, and mathematical physics (Potsdam, 1996), pp. 138-152, Math. Res. 100, Akademie, Berlin, 1997 Zbl 0889.35047 MR 1456184

[19] J. Ginibre and G. Velo, The Cauchy problem in local spaces for the complex Ginzburg-Landau equation. II. Contraction methods. Comm. Math. Phys. 187 (1997), no. 1, 45-79 Zbl 0889.35046 MR 1463822

[20] L. M. Hocking and K. Stewartson, On the nonlinear response of a marginally unstable plane parallel flow to a two-dimensional disturbance. Proc. Roy. Soc. London Ser. A 326 (1972), 289-313 Zbl 0228.76074 MR 329419

[21] L. M. Hocking, K. Stewartson, J. T. Stuart, and S. N. Brown, A nonlinear instability burst in plane parallel flow. J. Fluid. Mech. 51 (1972), 705-735 Zbl 0232.76053 
[22] P. Kolodner, D. Bensimon, and M. Surko, Traveling wave convection in an annulus. Phys. Rev. Lett. 60 (1988), 1723

[23] P. Kolodner, S. Slimani, N. Aubry, and R. Lima, Characterization of dispersive chaos and related states of binary-fluid convection. Phys. D 85 (1995), no. 1-2, 165-224

Zbl 0899.76019 MR 1339236

[24] N. Masmoudi and H. Zaag, Blow-up profile for the complex Ginzburg-Landau equation. J. Funct. Anal. 255 (2008), no. 7, 1613-1666 Zbl 1158.35016 MR 2442077

[25] F. Merle and H. Zaag, Stability of the blow-up profile for equations of the type $u_{t}=\Delta u+$ $|u|^{p-1} u$. Duke Math. J. 86 (1997), no. 1, 143-195 Zbl 0872.35049 MR 1427848

[26] V. T. Nguyen and H. Zaag, Construction of a stable blow-up solution for a class of strongly perturbed semilinear heat equations. Ann. Sc. Norm. Super. Pisa Cl. Sci. (5) 16 (2016), no. 4, 1275-1314 Zbl 1378.35178 MR 3616335

[27] N. Nouaili and H. Zaag, Profile for a simultaneously blowing up solution to a complex valued semilinear heat equation. Comm. Partial Differential Equations 40 (2015), no. 7, 1197-1217 Zbl 1335.35126 MR 3341202

[28] N. Nouaili and H. Zaag, Construction of a blow-up solution for the complex Ginzburg-Landau equation in a critical case. Arch. Ration. Mech. Anal. 228 (2018), no. 3, 995-1058

Zbl 1397.35295 MR 3780144

[29] P. Plecháč and V. Šverák, On self-similar singular solutions of the complex Ginzburg-Landau equation. Comm. Pure Appl. Math. 54 (2001), no. 10, 1215-1242 Zbl 1032.35162 MR 1843986

[30] S. Popp, O. Stiller, E. Kuznetsov, and L. Kramer, The cubic complex Ginzburg-Landau equation for a backward bifurcation. Phys. D 114 (1998), no. 1-2, 81-107 Zbl 0934.35176 MR 1612047

[31] P. Raphaël and R. Schweyer, Stable blowup dynamics for the 1-corotational energy critical harmonic heat flow. Comm. Pure Appl. Math. 66 (2013), no. 3, 414-480 Zbl 1270.35136 MR 3008229

[32] P. Raphaël and R. Schweyer, On the stability of critical chemotactic aggregation. Math. Ann. 359 (2014), no. 1-2, 267-377 Zbl 1320.35100 MR 3201901

[33] V. Rottschäfer, Multi-bump, self-similar, blow-up solutions of the Ginzburg-Landau equation. Phys. D 237 (2008), no. 4, 510-539 Zbl 1166.35376 MR 2451604

[34] V. Rottschäfer, Asymptotic analysis of a new type of multi-bump, self-similar, blowup solutions of the Ginzburg-Landau equation. European J. Appl. Math. 24 (2013), no. 1, 103-129 Zbl 1278.35236 MR 3041705

[35] K. Stewartson and J. T. Stuart, A non-linear instability theory for a wave system in plane Poiseuille flow. J. Fluid Mech. 48 (1971), 529-545 Zbl 0222.76045 MR 309420

[36] S. Tayachi and H. Zaag, Existence of a stable blow-up profile for the nonlinear heat equation with a critical power nonlinear gradient term. Trans. Amer. Math. Soc. 371 (2019), no. 8, 5899-5972 Zbl 1423.35186 MR 3937314

[37] J. J. L. Velázquez, V. A. Galaktionov, and M. A. Herrero, The space structure near a blow-up point for semilinear heat equations: a formal approach. Zh. Vychisl. Mat. i Mat. Fiz. 31 (1991), no. 3, 399-411 Zbl 0747.35014 MR 1107061

[38] H. Zaag, Blow-up results for vector-valued nonlinear heat equations with no gradient structure. Ann. Inst. H. Poincaré Anal. Non Linéaire 15 (1998), no. 5, 581-622 Zbl 0902.35050 MR 1643389

[39] H. Zaag, A Liouville theorem and blowup behavior for a vector-valued nonlinear heat equation with no gradient structure. Comm. Pure Appl. Math. 54 (2001), no. 1, 107-133

Zbl 1029.35113 MR 1787109 
Received 25 November 2020; accepted 31 March 2021.

\section{Giao Ky Duong}

Department of Mathematics, New York University in Abu Dhabi, Saadiyat Island, P.O. Box 129188, Abu Dhabi, United Arab Emirates; gkd1@ nyu.edu

\section{Nejla Nouaili}

CEREMADE, Université Paris Dauphine, Paris Sciences et Lettres, Place du Maréchal de Lattre de Tassigny, 75775 Paris Cedex 16, France; Nejla.Nouaili@dauphine.fr

\section{Hatem Zaag}

LAGA, CNRS (UMR 7539), Université Paris 13, Sorbonne Paris Cité, 99 avenue Jean-Baptiste Clément, 93430 Villetaneuse, France; Hatem.Zaag@univ-paris13.fr 